\title{
Unitary Processes with Independent Increments and Representations of Hilbert Tensor Algebras
}

\author{
By \\ Lingaraj SAHU*, Michael SchÜRmanN** and Kalyan B. SinhA***
}

\begin{abstract}
The aim of this article is to characterize unitary increment process by a quantum stochastic integral representation on symmetric Fock space. Under certain assumptions we have proved its unitary equivalence to a Hudson-Parthasarathy flow.
\end{abstract}

\section{$\S 1$. Introduction}

In the framework of the theory of quantum stochastic calculus developed by pioneering work of Hudson and Parthasarathy [6], quantum stochastic differential equations (qsde) of the form

$$
d V_{t}=\sum_{\mu, \nu \geq 0} V_{t} L_{\nu}^{\mu} \Lambda_{\mu}^{\nu}(d t), V_{0}=1_{\mathbf{h} \otimes \Gamma}
$$

(where the coefficients $L_{\nu}^{\mu}: \mu, \nu \geq 0$ are operators in the initial Hilbert space $\mathbf{h}$ and $\Lambda_{\mu}^{\nu}$ are fundamental processes in the symmetric Fock space $\Gamma=$ $\Gamma_{\text {sym }}\left(L^{2}\left(\mathbb{R}_{+}, \mathbf{k}\right)\right)$ with respect to a fixed orthonormal basis (in short 'ONB')

Communicated by Y. Takahashi. Received January 7, 2008. Revised December 2, 2008. 2000 Mathematics Subject Classification(s): 81S25, 47D03, 60 G51.

* Stat-Math Unit, Indian Statistical Institute, Bangalore Centre, $8^{\text {th }}$ Mile, Mysore Road, Bangalore-59, India.

e-mail: lingaraj@gmail.com

**Institut für Mathematik und Informatik, F.-L.-Jahn-Strasse 15a, D-17487 Greifswald, Germany.

e-mail: schurman@uni-greifswald.de

*** Jawaharlal Nehru Centre for Advanced Scientific Research, Jakkur, Bangalore-64, and Department of Mathematics, Indian Institute of Science, Bangalore-12, India.

e-mail: kbs_jaya@yahoo.co.in

(C) 2009 Research Institute for Mathematical Sciences, Kyoto University. All rights reserved. 
$\left\{E_{j}: j \geq 1\right\}$ of the noise Hilbert space $\mathbf{k}$ ) have been formulated. Conditions for existence and uniqueness of a solution $\left\{V_{t}\right\}$ are studied by Hudson and Parthasarathy and many other authors. In particular when the coefficients $L_{\nu}^{\mu}: \mu, \nu \geq 0$ are bounded operators satisfying some conditions it is observed that the solution $\left\{V_{t}: t \geq 0\right\}$ is a unitary process.

In [4], using the integral representation of regular quantum martingales in symmetric Fock space [17], the authors show that any covariant Fock adapted unitary evolution $\left\{V_{s, t}: 0 \leq s \leq t<\infty\right\}$ (with norm-continuous expectation semigroup) satisfies a quantum stochastic differential equation (1.1) with constant coefficients $L_{\nu}^{\mu} \in \mathcal{B}(\mathbf{h})$. For situations where the expectation semigroup is not norm continuous, the characterization problem is discussed in $[5,1]$. In $[10,11]$, by extended semigroup methods, Lindsay and Wills have studied such problems for Fock adapted contractive operator cocycles and completely positive cocyles.

In this article we are interested in the characterization of unitary evolutions with stationary and independent increments on $\mathbf{h} \otimes \mathcal{H}$, where $\mathbf{h}$ and $\mathcal{H}$ are separable Hilbert spaces. In $[18,19]$, by a co-algebraic treatment, the second author has proved that any weakly continuous unitary stationary independent increment process on $\mathbf{h} \otimes \mathcal{H}, \mathbf{h}$ finite dimensional, is unitarily equivalent to a Hudson-Parthasarathy flow with constant operator coefficients; see also $[8,9]$. In this present paper we treat the case of a unitary stationary independent increment process on $\mathbf{h} \otimes \mathcal{H}, \mathbf{h}$ not necessarily finite dimensional, with normcontinuous expectation semigroup. By a GNS type construction we are able to get the noise space $\mathbf{k}$ and the bounded operator coefficients $L_{\nu}^{\mu}$ such that the Hudson-Parthasarathy flow equation (1.1) admits a unique unitary solution and is unitarily equivalent to the unitary process we started with.

The article is organized as follows: Section 2 is meant for recalling some preliminary ideas and fixing some notations on linear operators on Hilbert spaces and quantum stochastic flows on Fock space. In the next Section an algebra structure is given on tensor product of Hilbert space which we are calling as Hilbert tensor algebra. The unitary processes with stationary and independent increments are described in Section 4 and filtration property of these processes is seen in Section 5. In Section 6 various semigroups associated with above mentioned unitary processes are studied and using them a Hilbert space, called noise space and structure maps are constructed from the Hilbert tensor algebra in Section 7. Associated Hudson-Parthasarathy flow is studied in Section 8 and its minimality is discussed in Section 9. In the last Section unitary equivalence to Hudson-Parthasarathy flow is established. 


\section{$\S 2 . \quad$ Notation and Preliminaries}

We assume that all the Hilbert spaces appearing in this article are complex separable with inner product anti-linear in the first variable. For any Hilbert spaces $\mathcal{H}, \mathcal{K} \quad \mathcal{B}(\mathcal{H}, \mathcal{K})$ and $\mathcal{B}_{1}(\mathcal{H})$ denote the Banach space of bounded linear operators from $\mathcal{H}$ to $\mathcal{K}$ and trace class operators on $\mathcal{H}$ respectively. For a linear (not necessarily bounded) map $T$ we write its domain as $\mathcal{D}(T)$. We denote the trace on $\mathcal{B}_{1}(\mathcal{H})$ by $\operatorname{Tr}_{\mathcal{H}}$ or simply $\operatorname{Tr}$. The von Neumann algebra of bounded linear operators on $\mathcal{H}$ is denoted by $B(\mathcal{H})$. The Banach space $\mathcal{B}_{1}(\mathcal{H}, \mathcal{K}) \equiv\{\rho \in$ $\left.\mathcal{B}(\mathcal{H}, \mathcal{K}):|\rho|:=\sqrt{\rho^{*} \rho} \in \mathcal{B}_{1}(\mathcal{H})\right\}$ with norm (Ref. Page no. 47 in [2])

$\|\rho\|_{1}=\||\rho|\|_{\mathcal{B}_{1}(\mathcal{H})}=\sup \left\{\sum_{k, l}\left|\left\langle\phi_{k}, \rho \psi_{l}\right\rangle\right|:\left\{\phi_{k}\right\},\left\{\psi_{l}\right\}\right.$ are $O N B$ of $\mathcal{K}$ and $\mathcal{H}$ resp. $\}$

is the predual of $\mathcal{B}(\mathcal{K}, \mathcal{H})$. For an element $x \in \mathcal{B}(\mathcal{K}, \mathcal{H}), \quad \mathcal{B}_{1}(\mathcal{H}, \mathcal{K}) \ni \rho \mapsto$ $\operatorname{Tr}_{\mathcal{H}}(x \rho)$ defines an element of the dual Banach space $\mathcal{B}_{1}(\mathcal{H}, \mathcal{K})^{*}$. For a linear map $T$ on the Banach space $\mathcal{B}_{1}(\mathcal{H}, \mathcal{K})$ the adjoint $T^{*}$ on the dual $\mathcal{B}(\mathcal{K}, \mathcal{H})$ is given by $\operatorname{Tr}_{\mathcal{H}}\left(T^{*}(x) \rho\right):=\operatorname{Tr}_{\mathcal{H}}(x T(\rho)), \forall x \in \mathcal{B}(\mathcal{K}, \mathcal{H}), \rho \in \mathcal{B}_{1}(\mathcal{H}, \mathcal{K})$.

For any $\xi \in \mathcal{H} \otimes \mathcal{K}, h \in \mathcal{H}$ the map

$$
\mathcal{K} \ni k \mapsto\langle\xi, h \otimes k\rangle
$$

defines a bounded linear functional on $\mathcal{K}$ and thus by Riesz's theorem there exists a unique vector $\langle\langle\xi, h\rangle\rangle$ in $\mathcal{K}$ such that

$$
\langle\langle\langle\xi, h\rangle\rangle, k\rangle=\langle\xi, h \otimes k\rangle, \forall k \in \mathcal{K} .
$$

In other words $\langle\langle\xi, h\rangle\rangle=F_{h}^{*} \xi$ where $F_{h} \in \mathcal{B}(\mathcal{K}, \mathcal{H} \otimes \mathcal{K})$ is given by $F_{h} k=h \otimes k$.

Let $\mathbf{h}$ and $\mathcal{H}$ be two Hilbert spaces with some orthonormal bases $\left\{e_{j}: j \geq\right.$ $1\}$ and $\left\{\zeta_{n}: n \geq 1\right\}$ respectively. For $A \in \mathcal{B}(\mathbf{h} \otimes \mathcal{H})$ and $u, v \in \mathbf{h}$ we define a linear operator $A(u, v) \in \mathcal{B}(\mathcal{H})$ by

$$
\left\langle\xi_{1}, A(u, v) \xi_{2}\right\rangle=\left\langle u \otimes \xi_{1}, A v \otimes \xi_{2}\right\rangle, \forall \xi_{1}, \xi_{2} \in \mathcal{H}
$$

and read off the following properties:

Lemma 2.1. Let $A, B \in \mathcal{B}(\mathbf{h} \otimes \mathcal{H})$ then for any $u, v, u_{i}$ and $v_{i}, i=1,2$ in $\mathbf{h}$

(i) $A(u, v) \in \mathcal{B}(\mathcal{H})$ with $\|A(u, v)\| \leq\|A\|\|u\|\|v\|$ and $A(u, v)^{*}=A^{*}(v, u)$.

(ii) $\mathbf{h} \times \mathbf{h} \mapsto A(\cdot, \cdot)$ is $1-1$, i.e. if $A(u, v)=B(u, v), \forall u, v \in \mathbf{h}$ then $A=B$. 
(iii) $A\left(u_{1}, v_{1}\right) B\left(u_{2}, v_{2}\right)=\left[A\left(\left|v_{1}><u_{2}\right| \otimes 1_{\mathcal{H}}\right) B\right]\left(u_{1}, v_{2}\right)$

(iv) $A B(u, v)=\sum_{j \geq 1} A\left(u, e_{j}\right) B\left(e_{j}, v\right)$ (strongly)

(v) $0 \leq A(u, v)^{*} A(u, v) \leq\|u\|^{2} A^{*} A(v, v)$

(vi) $\left\langle A(u, v) \xi_{1}, B(p, w) \xi_{2}\right\rangle=\sum_{n \geq 1}\left\langle p \otimes \zeta_{n},\left[B\left(|w><v| \otimes\left|\xi_{2}><\xi_{1}\right|\right) A^{*} u \otimes \zeta_{n}\right\rangle\right.$ $=\left\langle v \otimes \xi_{1},\left[A^{*}\left(|u><p| \otimes 1_{\mathcal{H}}\right) B w \otimes \xi_{2}\right\rangle\right.$.

Proof. We are omitting the proof of (i), (ii).

(iii) For any $\xi, \zeta \in \mathcal{H}$ we have

$$
\begin{aligned}
\left\langle\xi, A\left(u_{1}, v_{1}\right) B\left(u_{2}, v_{2}\right) \zeta\right\rangle & =\left\langle u_{1} \otimes \xi, A v_{1} \otimes B\left(u_{2}, v_{2}\right) \zeta\right\rangle \\
& =\left\langle A^{*} u_{1} \otimes \xi, v_{1} \otimes B\left(u_{2}, v_{2}\right) \zeta\right\rangle \\
& =\sum_{n \geq 1}\left\langle A^{*} u_{1} \otimes \xi, v_{1} \otimes \zeta_{n}\right\rangle\left\langle\zeta_{n}, B\left(u_{2}, v_{2}\right) \zeta\right\rangle \\
& =\sum_{n \geq 1}\left\langle A^{*} u_{1} \otimes \xi, v_{1} \otimes \zeta_{n}\right\rangle\left\langle u_{2} \otimes \zeta_{n}, B v_{2} \otimes \zeta\right\rangle \\
& =\sum_{n \geq 1}\left\langle A^{*} u_{1} \otimes \xi,\left(\mid v_{1}>\left\langle u_{2}|\otimes| \zeta_{n}>\left\langle\zeta_{n}\right|\right) B v_{2} \otimes \zeta\right\rangle\right. \\
& =\left\langle u_{1} \otimes \xi, A\left(\left|v_{1}><u_{2}\right| \otimes 1_{\mathcal{H}}\right) B v_{2} \otimes \zeta\right\rangle .
\end{aligned}
$$

Thus it follows that

$$
A\left(u_{1}, v_{1}\right) B\left(u_{2}, v_{2}\right)=\left[A\left(\left|v_{1}><u_{2}\right| \otimes 1_{\mathcal{H}}\right) B\right]\left(u_{1}, v_{2}\right) .
$$

(iv) By part (iii)

$$
\begin{aligned}
& \sum_{j=1}^{N}\left\|A\left(e_{j}, u\right) \xi\right\|^{2} \\
& =\sum_{j=1}^{N}\left\langle\xi, A^{*}\left(u, e_{j}\right) A\left(e_{j}, u\right) \xi\right\rangle \\
& =\left\langle\xi,\left[A^{*}\left(P_{N} \otimes 1_{\mathcal{H}}\right) A\right](u, u) \xi\right\rangle,
\end{aligned}
$$

where $P_{N}$ is the finite rank projection $\sum_{j=1}^{N}\left|e_{j}><e_{j}\right|$ on $\mathbf{h}$. Since $\left\{\left[A^{*}\left(P_{N} \otimes\right.\right.\right.$ $\left.\left.\left.1_{\mathcal{H}}\right) A\right](u, u)\right\}$ is an increasing sequence of positive operators and $0 \leq P_{N} \otimes 1_{\mathcal{H}}$ converges strongly to $1_{\mathbf{h} \otimes \mathcal{H}}$ as $N$ tends to $\infty,\left[A^{*}\left(P_{N} \otimes 1_{\mathcal{H}}\right) A\right](u, u)$ converges strongly to $\left[A^{*} A\right](u, u)$ as $N$ tends to $\infty$. Thus

$$
\lim _{N \rightarrow \infty} \sum_{j=1}^{N}\left\|A\left(e_{j}, u\right) \xi\right\|^{2}=\left\langle\xi,\left[A^{*} A\right](u, u) \xi\right\rangle
$$


and

$$
\sum_{j=1}^{N}\left\|A\left(e_{j}, u\right) \xi\right\|^{2} \leq\|A u \otimes \xi\|^{2} \leq\|A\|^{2}\|u\|^{2}\|\xi\|^{2}, \forall N \geq 1 .
$$

Now let us consider the following, for $\xi, \zeta \in \mathcal{H}$

$$
\begin{aligned}
& \left|\left\langle\xi, \sum_{j=1}^{N} A\left(u, e_{j}\right) B\left(e_{j}, v\right) \zeta\right\rangle\right|^{2}=\left|\sum_{j=1}^{N}\left\langle A^{*}\left(e_{j}, u\right) \xi, B\left(e_{j}, v\right) \zeta\right\rangle\right|^{2} \\
& \leq \sum_{j=1}^{N}\left\|A^{*}\left(e_{j}, u\right) \xi\right\|^{2} \sum_{j=1}^{N}\left\|B\left(e_{j}, v\right) \zeta\right\|^{2} \\
& \leq\|A\|^{2}\|u\|^{2}\|\xi\|^{2}\|B\|^{2}\|v\|^{2}\|\zeta\|^{2} .
\end{aligned}
$$

So

$$
\left|\left\langle\xi, \sum_{j=1}^{N} A\left(u, e_{j}\right) B\left(e_{j}, v\right) \zeta\right\rangle\right| \leq\|A\|\|B\|\|u\|\|v\|\|\xi\|\|\zeta\|
$$

and strong convergence of $\sum_{j \geq 1} A\left(u, e_{j}\right) B\left(e_{j}, v\right)$ follows.

(v) We have

$$
\begin{aligned}
& \left\langle\xi, A(u, v)^{*} A(u, v) \xi\right\rangle=\sum_{n \geq 1}\left\langle\xi, A^{*}(v, u) \zeta_{n}\right\rangle\left\langle\zeta_{n}, A(u, v) \xi\right\rangle \\
& =\sum_{n \geq 1}\left\langle v \otimes \xi, A^{*} u \otimes \zeta_{n}\right\rangle\left\langle u \otimes \zeta_{n}, A v \otimes \xi\right\rangle \\
& =\left\langle v \otimes \xi, A^{*}\left\{|u><u| \otimes \sum_{n \geq 1}\left|\zeta_{n}><\zeta_{n}\right|\right\} A v \otimes \xi\right\rangle .
\end{aligned}
$$

Since $\sum_{n \geq 1}\left|\zeta_{n}><\zeta_{n}\right|$ converges strongly to the identity operator

$$
\left\langle\xi, A(u, v)^{*} A(u, v) \xi\right\rangle \leq\|u\|^{2}\left\langle v \otimes \xi, A^{*} A v \otimes \xi\right\rangle
$$

and this proves the result.

(vi) We have

$$
\begin{aligned}
& \left\langle A(u, v) \xi_{1}, B(p, w) \xi_{2}\right\rangle \\
& =\sum_{n \geq 1}\left\langle A(u, v) \xi_{1}, \zeta_{n}\right\rangle\left\langle\zeta_{n}, B(p, w) \xi_{2}\right\rangle \\
& =\sum_{n \geq 1}\left\langle A v \otimes \xi_{1}, u \otimes \zeta_{n}\right\rangle\left\langle p \otimes \zeta_{n}, B w \otimes \xi_{2}\right\rangle \\
& =\sum_{n \geq 1}\left\langle B^{*} p \otimes \zeta_{n}, w \otimes \xi_{2}\right\rangle\left\langle v \otimes \xi_{1}, A^{*} u \otimes \zeta_{n}\right\rangle \\
& =\sum_{n \geq 1}\left\langle p \otimes \zeta_{n}, B\left(|w><v| \otimes\left|\xi_{2}><\xi_{1}\right|\right) A^{*} u \otimes \zeta_{n}\right\rangle .
\end{aligned}
$$


This proves the first part. The other part follows from

$$
\begin{aligned}
& \sum_{n \geq 1}\left\langle p \otimes \zeta_{n}, B\left(|w><v| \otimes\left|\xi_{2}><\xi_{1}\right|\right) A^{*} u \otimes \zeta_{n}\right\rangle \\
& =\operatorname{Tr}_{\mathbf{h} \otimes \mathcal{H}}\left[\left(|u><p| \otimes 1_{\mathcal{H}}\right) B\left(|w><v| \otimes\left|\xi_{2}><\xi_{1}\right|\right) A^{*}\right] \\
& =\operatorname{Tr}_{\mathbf{h} \otimes \mathcal{H}}\left[\left(|w><v| \otimes\left|\xi_{2}><\xi_{1}\right|\right) A^{*}\left(|u><p| \otimes 1_{\mathcal{H}}\right) B\right] \\
& =\left\langle v \otimes \xi_{1},\left[A^{*}\left(|u><p| \otimes 1_{\mathcal{H}}\right) B w \otimes \xi_{2}\right\rangle\right.
\end{aligned}
$$

Let us briefly recall the fundamental integrator processes of quantum stochastic calculus and the flow equation, introduced by Hudson and Parthasarathy [6]. For a Hilbert space $\mathbf{k}$ let us consider the symmetric Fock space $\Gamma=\Gamma\left(L^{2}\left(\mathbb{R}_{+}, \mathbf{k}\right)\right)$. The exponential vector in the Fock space, associated with a vector $f \in L^{2}\left(\mathbb{R}_{+}, \mathbf{k}\right)$ is given by

$$
\mathbf{e}(f)=\bigoplus_{n \geq 0} \frac{1}{\sqrt{n !}} f^{(n)},
$$

where $f^{(n)}=\underbrace{f \otimes f \otimes \cdots \otimes f}_{n \text {-copies }}$ for $n>0$ and by convention $f^{(0)}=1$. The exponential vector $\mathbf{e}(0)$ is called the vacuum vector.

Let us consider the Hudson-Parthasarathy (HP) flow equation on $\mathbf{h} \otimes$ $\Gamma\left(L^{2}\left(\mathbb{R}_{+}, \mathbf{k}\right)\right)$ :

$$
V_{s, t}=1_{\mathbf{h} \otimes \Gamma}+\sum_{\mu, \nu \geq 0} \int_{s}^{t} V_{s, \tau} L_{\nu}^{\mu} \Lambda_{\mu}^{\nu}(d \tau) .
$$

Here the coefficients $L_{\nu}^{\mu}: \mu, \nu \geq 0$ are operators in $\mathbf{h}$ and $\Lambda_{\mu}^{\nu}$ are fundamental processes with respect to a fixed orthonormal basis $\left\{E_{j}: j \geq 1\right\}$ of $\mathbf{k}$ :

$$
\Lambda_{\nu}^{\mu}(t)= \begin{cases}t 1_{\mathbf{h} \otimes \Gamma} & \text { for }(\mu, \nu)=(0,0) \\ a\left(1_{[0, t]} \otimes E_{j}\right) & \text { for }(\mu, \nu)=(j, 0) \\ a^{\dagger}\left(1_{[0, t]} \otimes E_{k}\right) & \text { for }(\mu, \nu)=(0, k) \\ \Lambda\left(1_{[0, t]} \otimes\left|E_{k}><E_{j}\right|\right) & \text { for }(\mu, \nu)=(j, k) .\end{cases}
$$

These operators act non-trivially on $\Gamma_{[0, t]}$ and as identity on $\Gamma_{(t, \infty)}$, where for any interval $I \subseteq[0, \infty), \Gamma_{I}=\Gamma_{\text {sym }}\left(L^{2}(I)\right)$. For any $0 \leq s \leq t<\infty, \Gamma=$ $\Gamma_{[0, s]} \otimes \Gamma_{(s, t]} \otimes \Gamma_{(t, \infty)}$.

Theorem $2.2([7,14,16,3])$. Let $H \in \mathcal{B}(\mathbf{h})$ be self-adjoint, $\left\{L_{k}, W_{k}^{j}:\right.$ $j, k \geq 1\}$ be a family of bounded linear operators in $\mathbf{h}$ such that $W=\sum_{j, k \geq 1} W_{k}^{j} \otimes$ 
$\left|E_{j}><E_{k}\right|$ is an isometry (respectively co-isometry) operator in $\mathbf{h} \otimes \mathbf{k}$ and for some constant $c \geq 0$,

$$
\sum_{k \geq 1}\left\|L_{k} u\right\|^{2} \leq c\|u\|^{2}, \forall u \in \mathbf{h} .
$$

Let the coefficients $L_{\nu}^{\mu}$ be as follows,

$$
L_{\nu}^{\mu}= \begin{cases}i H-\frac{1}{2} \sum_{k \geq 1} L_{k}^{*} L_{k} & \text { for }(\mu, \nu)=(0,0) \\ L_{j} & \text { for }(\mu, \nu)=(j, 0) \\ -\sum_{j \geq 1} L_{j}^{*} W_{k}^{j} & \text { for }(\mu, \nu)=(0, k) \\ W_{k}^{j}-\delta_{k}^{j} & \text { for }(\mu, \nu)=(j, k) .\end{cases}
$$

Then there exists a unique isometry (respectively co-isometry) operator valued process $V_{s, t}$ satisfying $(2.2)$.

\section{$\S 3 . \quad$ Hilbert Tensor Algebra}

For a product vector $\underline{u}=u_{1} \otimes u_{2} \otimes \cdots \otimes u_{n} \in \mathbf{h}^{\otimes n}$ we shall denote the product vector $u_{n} \otimes u_{n-1} \otimes \cdots \otimes u_{1}$ by $\underline{u}$. For the null vector in $\mathbf{h}^{\otimes n}$ we shall write $\underline{0}$. If $\left\{f_{j}\right\}_{j=1}^{\infty}$ is an ONB for $\mathbf{h}$, then we have a product ONB $\left\{f_{\dot{j}}=f_{j_{1}} \otimes \cdots \otimes f_{j_{n}}: j=\left(j_{1}, j_{2}, \cdots, j_{n}\right), j_{k} \geq 1\right\}$ for the Hilbert space $\mathbf{h}^{\otimes n}$.

Consider $\mathbb{Z}_{2}=\{0,1\}$, the finite field with addition modulo 2. For $n \geq 1$, let $\mathbb{Z}_{2}^{n}$ denote the $n$-fold direct sum of $\mathbb{Z}_{2}$. For $\underline{\epsilon}=\left(\epsilon_{1}, \epsilon_{2}, \cdots, \epsilon_{n}\right)$ and $\underline{\epsilon}^{\prime}=$ $\left(\epsilon_{1}^{\prime}, \epsilon_{2}^{\prime}, \cdots, \epsilon_{m}^{\prime}\right)$ we define

$\underline{\epsilon} \oplus \underline{\epsilon}^{\prime}=\left(\epsilon_{1}, \cdots, \epsilon_{n}, \epsilon_{1}^{\prime}, \cdots, \epsilon_{m}^{\prime}\right) \in \mathbb{Z}_{2}^{n+m}$ and $\underline{\epsilon}^{*}=\left(1+\epsilon_{n}, 1+\epsilon_{n-1}, \cdots, 1+\epsilon_{1}\right) \in \mathbb{Z}_{2}^{n}$.

Let $A \in \mathcal{B}(\mathbf{h} \otimes \mathcal{H}), \epsilon \in \mathbb{Z}_{2}=\{0,1\}$. We define operators $A^{(\epsilon)} \in \mathcal{B}(\mathbf{h} \otimes \mathcal{H})$ by $A^{(\epsilon)}:=A$ if $\epsilon=0$ and $A^{(\epsilon)}:=A^{*}$ if $\epsilon=1$. For $1 \leq k \leq n$, we define a unitary exchange map $P_{k, n}: \mathbf{h}^{\otimes n} \otimes \mathcal{H} \rightarrow \mathbf{h}^{\otimes n} \otimes \mathcal{H}$ by putting

$$
P_{k, n}(\underline{u} \otimes \xi):=u_{1} \otimes \cdots \otimes u_{k-1} \otimes u_{k+1} \cdots \otimes u_{n} \otimes\left(u_{k} \otimes \xi\right)
$$

on product vectors. Let $\underline{\epsilon}=\left(\epsilon_{1}, \epsilon_{2}, \cdots, \epsilon_{n}\right) \in \mathbb{Z}_{2}^{n}$. Consider the ampliation of the operator $A^{\left(\epsilon_{k}\right)}$ in $\mathcal{B}\left(\mathbf{h}^{\otimes n} \otimes \mathcal{H}\right)$ given by

$$
A^{\left(n, \epsilon_{k}\right)}:=P_{k, n}^{*}\left(1_{\mathbf{h} \otimes(n-1)} \otimes A^{\left(\epsilon_{k}\right)}\right) P_{k, n} .
$$

By definition of unitary operators $P_{k, n}$ for product vectors $\underline{u}, \underline{v} \in \mathbf{h}^{\otimes n}$,

$$
\left\langle\xi, A^{\left(n, \epsilon_{k}\right)}(\underline{u}, \underline{u}) \xi^{\prime}\right\rangle=\left\langle\xi, A^{\left(\epsilon_{k}\right)}\left(u_{k}, v_{k}\right) \xi^{\prime}\right\rangle \prod_{j \neq k}^{n}\left\langle u_{j}, v_{j}\right\rangle, \forall \xi, \xi^{\prime} \in \mathbf{H} .
$$


Now we define the operator $A^{(\underline{\epsilon})}:=\prod_{k=1}^{n} A^{\left(n, \epsilon_{k}\right)}:=A^{\left(1, \epsilon_{1}\right)} \cdots A^{\left(n, \epsilon_{n}\right)}$ in $\mathcal{B}\left(\mathbf{h}^{\otimes n} \otimes \mathcal{H}\right)$. Please note that as here, through out this article, the product symbol $\prod_{k=1}^{n}$ stands for product from left to right as $k$ increases. We have the following preliminary observation.

Lemma 3.1. (i) For product vectors $\underline{u}, \underline{v} \in \mathbf{h}^{\otimes n}$

$$
\prod_{i=1}^{m} A^{\left(n, \epsilon_{i}\right)}(\underline{u}, \underline{v})=\prod_{i=1}^{m} A^{\epsilon_{i}}\left(u_{i}, v_{i}\right) \prod_{i=m+1}^{n}\left\langle u_{i}, v_{i}\right\rangle \in \mathcal{B}(\mathcal{H}) .
$$

(ii) For $\xi, \zeta \in \mathcal{H}$

$$
\prod_{i=1}^{m} A^{\left(\epsilon_{i}\right)}(\xi, \zeta)=A^{\left(\underline{\epsilon}^{(m)}\right)}(\xi, \zeta) \otimes 1_{\mathbf{h} \otimes n-m} \in B\left(\mathbf{h}^{\otimes n}\right) .
$$

(iii) If $A$ is an isometry (respectively unitary) then $A^{\left(n, \epsilon_{k}\right)}$ and $A^{(\underline{\epsilon})}$ are isometries (respectively unitaries).

The proof is obvious and is omitted.

We note that part (i) of this Lemma in particular gives

$$
A^{(\underline{\epsilon})}(\underline{u}, \underline{v})=\prod_{i=1}^{n} A^{\left(\epsilon_{i}\right)}\left(u_{i}, v_{i}\right)
$$

Let $M_{0}:=\left\{(\underline{u}, \underline{v}, \underline{\epsilon}): \underline{u}=\otimes_{i=1}^{n} u_{i}, \underline{v}=\otimes_{i=1}^{n} v_{i} \in \mathbf{h}^{\otimes n}, \underline{\epsilon}=\left(\epsilon_{1}, \epsilon_{2}, \cdots, \epsilon_{n}\right) \in\right.$ $\left.\mathbb{Z}_{2}^{n}, n \geq 1\right\}$. In $M_{0}$, we introduce an equivalence relation ' $\sim$ ': $(\underline{u}, \underline{v}, \underline{\epsilon}) \sim$ $\left(\underline{p}, \underline{w}, \underline{\epsilon}^{\prime}\right)$ if $\underline{\epsilon}=\underline{\epsilon}^{\prime}$ and $|\underline{u}><\underline{v}|=|\underline{p}><\underline{w}| \in \mathcal{B}\left(\mathbf{h}^{\otimes n}\right)$. Expanding the vectors in term of the ONB $\left\{e_{j}=e_{j_{1}} \otimes \cdots \otimes e_{j_{n}}: j=\left(j_{1}, j_{2}, \cdots, j_{n}\right), j_{k} \geq 1\right\}$, from $|\underline{u}><\underline{v}|=|\underline{p}><\underline{w}|$ we get $\underline{u}_{\mathrm{j}} \underline{\bar{w}}_{\underline{\mathrm{k}}}=\underline{p}_{\mathrm{j}} \underline{\bar{w}}_{\mathrm{k}}$ for each multi-indices $\underline{\mathrm{j}}, \underline{\mathrm{k}}$. Thus in particular when $(u, v, 0) \sim(p, \bar{w}, \overline{0})$, for any $\xi_{1}, \xi_{2} \in \mathcal{H}$ we have

$$
\begin{aligned}
& \left\langle\xi_{1}, A(u, v) \xi_{2}\right\rangle \\
& =\sum_{j, k \geq 1} \overline{u_{j}} v_{k}\left\langle e_{j} \otimes \xi_{1}, A e_{k} \otimes \xi_{2}\right\rangle \\
& =\sum_{j, k \geq 1} \overline{p_{j}} w_{k}\left\langle e_{j} \otimes \xi_{1}, A e_{k} \otimes \xi_{2}\right\rangle \\
& =\left\langle\xi_{1}, A(p, w) \xi_{2}\right\rangle .
\end{aligned}
$$

In fact $A(u, v)=A(p, w)$ iff $(u, v, 0) \sim(p, w, 0)$ and more generally $A^{(\underline{\epsilon})}(\underline{u}, \underline{v})=$ $A^{\left(\underline{\epsilon}^{\prime}\right)}(\underline{p}, \underline{w})$ iff $(\underline{u}, \underline{v}, \underline{\epsilon}) \sim\left(\underline{p}, \underline{w}, \underline{\epsilon}^{\prime}\right)$. It is easy to see that $(\underline{0}, \underline{v}, \underline{\epsilon}) \sim(\underline{u}, \underline{0}, \underline{\epsilon}) \sim$ $(\underline{0}, \underline{0}, \underline{\epsilon})$ and we call this class the 0 of the quotient set $M_{0} / \sim$. We first introduce the following operations on $M_{0}$ : 
Vector multiplication: $\quad(\underline{u}, \underline{v}, \underline{\epsilon}) \cdot\left(\underline{p}, \underline{w}, \underline{\epsilon}^{\prime}\right)=\left(\underline{u} \otimes \underline{p}, \underline{v} \otimes \underline{w}, \underline{\epsilon} \oplus \underline{\epsilon}^{\prime}\right)$ and Involution: $(\underline{u}, \underline{v}, \underline{\epsilon})^{*}=\left(\underline{v}, \underline{u}, \underline{\epsilon}^{*}\right)$.

Since $(\underline{u} \otimes \underline{p})=p_{m} \otimes \cdots \otimes p_{1} \otimes u_{n} \otimes \cdots \otimes u_{1}=(\underline{p} \otimes \underline{u})$ and $\left(\underline{\epsilon} \oplus \underline{\epsilon}^{\prime}\right)^{*}=\left(\underline{\epsilon}^{\prime}\right)^{*} \oplus \underline{\epsilon}^{*}$

$$
\begin{aligned}
& {\left[(\underline{u}, \underline{v}, \underline{\epsilon}) \cdot\left(\underline{p}, \underline{w}, \underline{\epsilon}^{\prime}\right)\right]^{*}=\left(\underline{u} \otimes \underline{p}, \underline{v} \otimes \underline{w}, \underline{\epsilon} \oplus \underline{\epsilon}^{\prime}\right)^{*}} \\
& =\left(\underline{v} \otimes \underline{w}, \underline{u} \otimes \underline{p},\left(\underline{\epsilon} \oplus \underline{\epsilon}^{\prime}\right)^{*}\right) \\
& =\left(\underline{w}^{\underline{w}} \otimes \underline{\underline{v}}, \underline{\underline{p}} \otimes \underline{u},\left(\underline{\epsilon}^{\prime}\right)^{*} \oplus \underline{\epsilon}^{*}\right) \\
& =\left(\underline{p}, \underline{w}, \underline{\epsilon}^{\prime}\right)^{*} \cdot(\underline{u}, \underline{v}, \underline{\epsilon})^{*} .
\end{aligned}
$$

It is clear that $\underline{\epsilon}=\underline{\epsilon}^{\prime} \Longrightarrow \underline{\epsilon}^{*}=\left(\underline{\epsilon}^{\prime}\right)^{*}$ and $|\underline{u}><\underline{v}|=|\underline{p}><\underline{w}|$ implies $|\underline{v}><\underline{u}|=|\underline{w}><\underline{p}|$. Thus $(\underline{u}, \underline{v}, \underline{\epsilon}) \sim\left(\underline{p}, \underline{w}, \underline{\epsilon}^{\prime}\right)$ implies $(\underline{u}, \underline{v}, \underline{\epsilon})^{*} \sim\left(\underline{p}, \underline{w}, \underline{\epsilon}^{\prime}\right)^{*}$. Moreover, $(\underline{u}, \underline{v}, \underline{\epsilon}) \sim\left(\underline{u}^{\prime}, \underline{v}^{\prime}, \underline{\epsilon}^{\prime}\right)$ and $(\underline{p}, \underline{w}, \underline{\alpha}) \sim\left(\underline{p}^{\prime}, \underline{w}^{\prime}, \underline{\alpha}^{\prime}\right)$ implies $\underline{\epsilon} \oplus \underline{\alpha}=\underline{\epsilon}^{\prime} \oplus \underline{\alpha}^{\prime}$ and $|\underline{u} \otimes \underline{p}><\underline{v} \otimes \underline{w}|=|\underline{u}><\underline{v}| \otimes|\underline{p}><\underline{w}|=\left|\underline{u}^{\prime}><\underline{v}^{\prime}\right| \otimes\left|\underline{p}^{\prime}><\underline{w}^{\prime}\right|=$ $\left|\underline{u}^{\prime} \otimes \underline{p}^{\prime}><\underline{v}^{\prime} \otimes \underline{w}^{\prime}\right|$. Thus involution and vector multiplication respect $\sim$.

Let $M$ be the complex vector space spanned by $M_{0} / \sim$. The elements of $M$ are formal finite linear combinations of elements of $M_{0} / \sim$. With the above multiplication and involution $M$ is a $*$-algebra.

\section{$\S 4 . \quad$ Unitary Processes with Stationary and Independent Increment}

Let $\left\{U_{s, t}: 0 \leq s \leq t<\infty\right\}$ be a family of unitary operators in $\mathcal{B}(\mathbf{h} \otimes \mathcal{H})$ and $\Omega$ be a fixed unit vector in $\mathcal{H}$. We shall also set $U_{t}:=U_{0, t}$ for simplicity. As we discussed in the previous section, let us consider the family of operators $\left\{U_{s, t}^{(\epsilon)}\right\}$ in $\mathcal{B}(\mathbf{h} \otimes \mathcal{H})$ for $\epsilon \in \mathbb{Z}_{2}$ given by $U_{s, t}^{(\epsilon)}=U_{s, t}$ if $\epsilon=0, U_{s, t}^{(\epsilon)}=U_{s, t}^{*}$ if $\epsilon=1$. Furthermore for $n \geq 1, \underline{\epsilon} \in \mathbb{Z}_{2}^{n}$ fixed, $1 \leq k \leq n$, we consider the families of operators $\left\{U_{s, t}^{\left(\epsilon_{k}\right)}\right\}$ and $\left\{U_{s, t}^{(\underline{\epsilon})}\right\}$ in $\mathcal{B}\left(\mathbf{h}^{\otimes n} \otimes \mathcal{H}\right)$. By Lemma 3.1 we observe that

$$
U_{s, t}^{(\underline{\epsilon})}(\underline{u}, \underline{v})=\prod_{i=1}^{n} U_{s, t}^{\left(\epsilon_{i}\right)}\left(u_{i}, v_{i}\right)
$$

For $\underline{\epsilon}=(0,0, \cdots, 0) \in \mathbb{Z}_{2}^{n}$ and $1 \leq k \leq n$, we shall write $U_{s, t}^{(n, k)}$ for the unitary operator $U_{s, t}^{\left(n, \epsilon_{k}\right)}$ and $U_{s, t}^{(n)}$ for the unitary $U_{s, t}^{(\epsilon)}$ on $\mathbf{h}^{\otimes n} \otimes \mathcal{H}$. For $n \geq 1, \underline{s}=$ $\left(s_{1}, s_{2}, \cdots, s_{n}\right), \underline{t}=\left(t_{1}, t_{2}, \cdots, t_{n}\right): 0 \leq s_{1} \leq t_{1} \leq s_{2} \leq \ldots \leq s_{n} \leq t_{n}<\infty$, $\underline{\epsilon}_{k}=\left(\alpha_{1}^{(k)}, \alpha_{2}^{(k)}, \cdots, \alpha_{m_{k}}^{(k)}\right) \in \mathbb{Z}_{2}^{m_{k}}: 1 \leq k \leq n, m=m_{1}+m_{2}+\cdots+m_{n}$ $\underline{\epsilon}=\underline{\epsilon}_{1} \oplus \underline{\epsilon}_{2} \oplus \cdots \oplus \underline{\epsilon}_{n} \in \mathbb{Z}_{2}^{m}$, we define $U_{\underline{s}, \underline{t}}^{\underline{\epsilon}} \in \mathcal{B}\left(\mathbf{h}^{\otimes m} \otimes \mathcal{H}\right)$ by setting

$$
U_{\underline{s}, \underline{t}}^{(\underline{\epsilon})}:=\prod_{k=1}^{n} U_{s_{k}, t_{k}}^{\left(\underline{\epsilon}_{k}\right)}
$$


Here $U_{s_{k}, t_{k}}^{\left(\underline{\epsilon}_{k}\right)}$ is looked upon as an operator in $\mathcal{B}\left(\mathbf{h}^{\otimes m} \otimes \mathcal{H}\right)$ by ampliation and appropriate tensor flip. So for $\underline{u}=\otimes_{k=1}^{n} \underline{u}_{k}, \underline{v}=\otimes_{k=1}^{n} \underline{v}_{k} \in \mathbf{h}^{\otimes m}$ we have

$$
U_{\underline{s}, \underline{t}}^{(\underline{\epsilon})}(\underline{u}, \underline{v})=\prod_{k=1}^{n} U_{s_{k}, t_{k}}^{\left(\underline{\epsilon}_{k}\right)}\left(\underline{u}_{k}, \underline{v}_{k}\right) .
$$

When there can be no confusion, for $\underline{\epsilon}=(0,0, \cdots, 0)$ we write $U_{\underline{s}, \underline{t}}$ for $U_{\underline{s}, \underline{t}}^{(\underline{\epsilon}}$. For $a, b \geq 0, \underline{s}=\left(s_{1}, s_{2}, \cdots, s_{n}\right), \underline{t}=\left(t_{1}, t_{2}, \cdots, t_{n}\right)$ we write $a \leq \underline{s}, \underline{t} \leq \underline{b}$ if $a \leq s_{1} \leq t_{1} \leq s_{2} \leq \ldots \leq s_{n} \leq t_{n} \leq b$.

Let us assume the following properties on the unitary family $U_{s, t}$ to establish unitary equivalence of $U_{s, t}$ with an HP flow.

\section{Assumption A}

A1 (Evolution) For any $0 \leq r \leq s \leq t<\infty, U_{r, s} U_{s, t}=U_{r, t}$.

A2 (Independence of increments) For any $0 \leq s_{i} \leq t_{i}<\infty: i=1,2$ such that $\left[s_{1}, t_{1}\right) \cap\left[s_{2}, t_{2}\right)=\varnothing$

(a) For every $u_{i}, v_{i} \in \mathbf{h}, U_{s_{1}, t_{1}}\left(u_{1}, v_{1}\right)$ commutes with $U_{s_{2}, t_{2}}\left(u_{2}, v_{2}\right)$ and $U_{s_{2}, t_{2}}^{*}\left(u_{2}, v_{2}\right)$.

(b) For $s_{1} \leq \underline{a}, \underline{b} \leq t_{1}, \quad s_{2} \leq \underline{q}, \underline{r} \leq t_{2}$ and $\underline{u}, \underline{v} \in \mathbf{h}^{\otimes n}, \underline{p}, \underline{w} \in \mathbf{h}^{\otimes m}$, $\underline{\epsilon} \in \mathbb{Z}_{2}^{n}, \underline{\epsilon}^{\prime} \in \mathbb{Z}_{2}^{m}$

$$
\left\langle\Omega, U_{\underline{a}, \underline{b}}^{(\underline{\epsilon})}(\underline{u}, \underline{v}) U_{\underline{q}, \underline{r}}^{\left(\underline{\epsilon}^{\prime}\right)}(\underline{p}, \underline{w}) \Omega\right\rangle=\left\langle\Omega, U_{\underline{a}, \underline{b}}^{(\underline{\epsilon})}(\underline{u}, \underline{v}) \Omega\right\rangle\left\langle\Omega, U_{\underline{q}, \underline{r}}^{\left(\underline{\epsilon}^{\prime}\right)}(\underline{p}, \underline{w}) \Omega\right\rangle .
$$

A3 (Stationarity) For any $0 \leq s \leq t<\infty$ and $\underline{u}, \underline{v} \in \mathbf{h}^{\otimes n}, \underline{\epsilon} \in \mathbb{Z}_{2}^{n}$

$$
\left\langle\Omega, U_{s, t}^{(\underline{\epsilon})}(\underline{u}, \underline{v}) \Omega\right\rangle=\left\langle\Omega, U_{t-s}^{(\underline{\epsilon})}(\underline{u}, \underline{v}) \Omega\right\rangle .
$$

\section{Assumption B (Uniform continuity)}

$$
\lim _{t \rightarrow 0} \sup \left\{\left|\left\langle\Omega,\left(U_{t}-1\right)(u, v) \Omega\right\rangle\right|:\|u\|,\|v\|=1\right\}=0 .
$$

Assumption C (Gaussian Condition) For any $u_{i}, v_{i} \in \mathbf{h}, \epsilon_{i} \in \mathbb{Z}_{2}: i=$ $1,2,3$

(4.2) $\lim _{t \rightarrow 0} \frac{1}{t}\left\langle\Omega,\left(U_{t}^{\left(\epsilon_{1}\right)}-1\right)\left(u_{1}, v_{1}\right)\left(U_{t}^{\left(\epsilon_{2}\right)}-1\right)\left(u_{2}, v_{2}\right)\left(U_{t}^{\left(\epsilon_{3}\right)}-1\right)\left(u_{3}, v_{3}\right) \Omega\right\rangle=0$.

\section{Assumption D (Minimality)}

The set $\mathcal{S}=\left\{U_{\underline{s}, \underline{t}} \underline{u}, \underline{v}\right) \Omega:=U_{s_{1}, t_{1}}\left(u_{1}, v_{1}\right) \cdots U_{s_{n}, t_{n}}\left(u_{n}, v_{n}\right) \Omega: \underline{s}=\left(s_{1}, s_{2}\right.$, $\left.\cdots, s_{n}\right), \underline{t}=\left(t_{1}, t_{2}, \cdots, t_{n}\right): 0 \leq s_{1} \leq t_{1} \leq s_{2} \leq \ldots \leq s_{n} \leq t_{n}<\infty, n \geq$ $\left.1, \underline{u}=\otimes_{i=1}^{n} u_{i}, \underline{v}=\otimes_{i=1}^{n} v_{i} \in \mathbf{h}^{\otimes n}\right\}$ is total in $\mathcal{H}$. 
Remark 4.1. (a) The Assumptions A, $\mathbf{B}$ and $\mathbf{C}$ hold in many situations, for example for unitary solutions of the Hudson-Parthasarathy flow (2.2) with bounded operator coefficients and having no Poisson terms. We will see (Lemma 6.6) that Assumption C means that expressions of the form (4.2) vanish for arbitrary length $n \geq 3$ not only for length 3 . This corresponds to the fact that the increments of order $n \geq 3$ of a Gaussian distribution vanish. Moreover, this property characterizes Gaussian distributions.

In the case of $\operatorname{dim}(\mathbf{h})<\infty$ Assumption $\mathbf{C}$ is equivalent to the condition that the generator of the quantum Lévy process associated with $\left\{U_{s, t}\right\}$ vanishes on products of length 3 of elements of the kernel of the counit. This again is equivalent to the condition that the preservation term in the corresponding quantum stochastic differential equation does not appear, Ref. Chapter 5 of [19].

Remark 4.2. The Assumption $\mathbf{D}$ is not really a restriction, since one can as well work with replacing $\mathcal{H}$ by the span closure of $S$. Taking $0 \leq s_{1} \leq$ $t_{1} \leq s_{2} \leq \ldots \leq s_{n} \leq t_{n}<\infty$ in the definition of $S \subseteq \mathcal{H}$ is enough for totality of the set $S$ because : for $0 \leq r \leq s \leq t \leq \infty$, we have $\left.U_{r, t}(p, w)\right)=$ $\sum_{j} U_{r, s}\left(p, e_{j}\right) U_{s, t}\left(e_{j}, w\right)$. So if there are overlapping intervals $\left[s_{k}, t_{k}\right) \cap\left[s_{k+1}\right.$, $\left.t_{k+1}\right) \neq \varnothing$ then the vector $\xi=U_{\underline{s}, \underline{t}}(\underline{u}, \underline{v}) \Omega$ in $\mathcal{H}$ can be obtained as a vector in the closure of the linear span of $S$.

For any $n \geq 1$ we have the following useful observations.

Lemma 4.3. (i) For any $0 \leq r \leq s \leq t<\infty$,

$$
U_{r, t}^{(n, k)}=U_{r, s}^{(n, k)} U_{s, t}^{(n, k)} .
$$

(ii) For any $1 \leq k_{1}, k_{2}, \cdots, k_{m} \leq n: k_{i} \neq k_{j}$ for $i \neq j$ and $0 \leq s_{i} \leq t_{i}<\infty$ : $i=1,2, \cdots, n$

$$
\prod_{i=1}^{m} U_{s_{i}, t_{i}}^{\left(n, \epsilon_{k_{i}}\right)}(\underline{u}, \underline{v})=\prod_{i=1}^{m} U_{s_{i}, t_{i}}^{\left(n, \epsilon_{k_{i}}\right)}\left(u_{k_{i}}, v_{k_{i}}\right) \prod_{j \neq k_{i}}\left\langle u_{j}, v_{j}\right\rangle
$$

for every $\underline{u}=\otimes_{i=1}^{n} u_{i}, \underline{v}=\otimes_{i=1}^{n} v_{i} \in \mathbf{h}^{\otimes n}$ and $\underline{\epsilon} \in \mathbb{Z}_{2}^{n}$.

(iii)

$$
U_{r, t}^{(n)}=U_{r, s}^{(n)} U_{s, t}^{(n)}
$$


Proof. (i) It follows from the definition and Assumptions A1, A2.

(ii) For $\underline{u}=\otimes_{i=1}^{n} u_{i}, \underline{v}=\otimes_{i=1}^{n} v_{i} \in \mathbf{h}^{\otimes n}$ and $\underline{\epsilon} \in \mathbb{Z}_{2}^{n}$ we can see by induction that

$$
\left\langle\xi, \prod_{i=1}^{m} U_{s_{i}, t_{i}}^{\left(n, \epsilon_{k_{i}}\right)}(\underline{u}, \underline{v}) \xi^{\prime}\right\rangle=\left\langle\xi, \prod_{i=1}^{m} U_{s_{i}, t_{i}}^{\left(n, \epsilon_{k_{i}}\right)}\left(u_{k_{i}}, v_{k_{i}}\right) \prod_{j \neq k_{i}} \xi^{\prime}\right\rangle \prod_{j \neq k}^{n}\left\langle u_{j}, v_{j}\right\rangle, \forall \xi, \xi^{\prime} \in \mathbf{H} .
$$

Thus (4.4) follows.

(iii) Since $U_{r, t}^{(n)}$ is a product of $U_{r, t}^{(n, k)}: k=1,2, \ldots, n$ and we have

$$
U_{r, t}^{(n, k)}=U_{r, s}^{(n, k)} U_{s, t}^{(n, k)},
$$

it is enough to prove that the unitary operators $U_{r, s}^{(n, k)}$ and $U_{s, t}^{(n, l)}$ commute for $k \neq l$. To see this let us consider the following. By part (ii) and the fact that $U_{r, s}\left(u_{k}, v_{k}\right)$ and $U_{s, t}\left(u_{l}, v_{l}\right)$ commute by Assumption A2, we get

$$
\begin{aligned}
& U_{r, s}^{(n, k)} U_{s, t}^{(n, l)}(\underline{u}, \underline{v})=U_{r, s}\left(u_{k}, v_{k}\right) U_{s, t}\left(u_{l}, v_{l}\right) \prod_{i \neq k, l}\left\langle u_{i}, v_{i}\right\rangle \\
& =U_{s, t}\left(u_{l}, v_{l}\right) U_{r, s}\left(u_{k}, v_{k}\right) \prod_{i \neq k, l}\left\langle u_{i}, v_{i}\right\rangle=U_{s, t}^{(n, l)} U_{r, s}^{(n, k)}(\underline{u}, \underline{v}) .
\end{aligned}
$$

As all the operators $U$ appear here are bounded this implies

$$
U_{r, s}^{(n, k)} U_{s, t}^{(n, l)}=U_{s, t}^{(n, l)} U_{r, s}^{(n, k)}
$$

\section{$\S 5 . \quad$ Filtration}

For any $0 \leq q \leq t<\infty$, let $\mathcal{H}_{[q, t]}=\overline{\operatorname{Span}} \mathcal{S}_{[q, t]}$, where $\mathcal{S}_{[q, t]} \subseteq \mathcal{H}$ is given by $\left\{\xi_{[q, t]}=U_{\underline{r}, \underline{s}}^{(n)}(\underline{u}, \underline{v}) \Omega=U_{r_{1}, s_{1}}\left(u_{1}, v_{1}\right) \cdots U_{r_{n}, s_{n}}\left(u_{n}, v_{n}\right) \Omega \in \mathcal{S}: q \leq \underline{r}, \underline{s}<\right.$ $\left.t, n \geq 1, \underline{u}, \underline{v} \in \underline{\mathbf{h}}^{\otimes n}\right\}$. We shall denote the Hilbert spaces $\mathcal{H}_{[0, t]}$ and $\mathcal{H}_{[t, \infty)}$ by $\mathcal{H}_{t]}$ and $\mathcal{H}_{[t}$ respectively.

Lemma 5.1. For $0 \leq t \leq T \leq \infty$, there exist a unitary isomorphism $\Xi_{t}: \mathcal{H}_{t]} \otimes \mathcal{H}_{(t, T]} \rightarrow \mathcal{H}_{T]}$ such that

$$
U_{t}(u, v)=\Xi_{t}^{*} U_{t}(u, v) \otimes 1_{\mathcal{H}_{(t, T]}} \Xi_{t} .
$$

Proof. Let us define a map $\Xi_{t}: \mathcal{H}_{t]} \otimes \mathcal{H}_{[t, T]} \rightarrow \mathcal{H}_{T]}$ by

$$
\Xi_{t}\left(\xi_{[0, t]} \otimes \zeta_{[t, T]}\right)=U_{\underline{r}, \underline{s}}^{(n)}(\underline{u}, \underline{v}) U_{\underline{r}^{\prime}, \underline{s}^{\prime}}^{(n)}(\underline{p}, \underline{w}) \Omega
$$

for $\xi_{[0, t]}=U_{\underline{r}, \underline{s}}^{(n)}(\underline{u}, \underline{v}) \Omega \in \mathcal{S}_{t]}$ and $\zeta_{[t, T]}=U_{\underline{r}^{\prime}, \underline{s}^{\prime}}^{(n)}(\underline{p}, \underline{w}) \Omega \in \mathcal{S}_{[t, T]}$, then extending linearly. 
Now let us consider the following. By Assumption A, for $\xi_{[0, t]}$ and $\zeta_{[t, T]}$ as above and $\eta_{[0, t]}=U_{\underline{a}, \underline{b}}^{(n)}(\underline{\mathrm{x}}, \underline{\mathrm{y}}) \Omega \in \mathcal{S}_{t]}$ and $\left.\gamma_{[t, T]}=U_{\underline{a}^{\prime}, \underline{b^{\prime}}}^{(\mathrm{g})}, \underline{\mathrm{h}}\right) \Omega \in \mathcal{S}_{[t, T]}$, we have

$$
\begin{aligned}
& \left\langle\Xi_{t}\left(\xi_{[0, t]} \otimes \zeta_{[t, T]}\right), \Xi_{t}\left(\eta_{[0, t]} \otimes \gamma_{[t, T]}\right)\right\rangle \\
& =\left\langle U_{\underline{r}, \underline{s}}^{(n)}(\underline{u}, \underline{v}) U_{\underline{\underline{r}}^{\prime}, \underline{\underline{S}}^{\prime}}^{(n)}(\underline{p}, \underline{w}) \Omega, U_{\underline{a}, \underline{b}}^{(n)}(\underline{\mathrm{x}}, \underline{\mathrm{y}}) U_{\underline{a}^{\prime}, \underline{b^{\prime}}}^{(\mathrm{n})}(\mathrm{g}, \underline{\mathrm{h}}) \Omega\right\rangle
\end{aligned}
$$

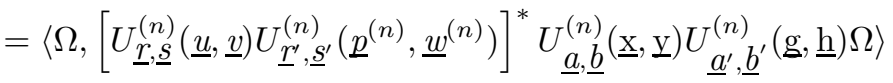

$$
\begin{aligned}
& \left.=\left\langle\Omega,\left[U_{\underline{r}, \underline{s}}^{(n)}(\underline{u}, \underline{v})\right]^{*} U_{\underline{a}, \underline{b}}^{(n)} \underline{\mathrm{x}}, \underline{\mathrm{y}}\right) \Omega\right\rangle \\
& \left\langle\Omega,\left[U_{\underline{r}^{\prime}, \underline{s}^{\prime}}^{(n)}(\underline{p}, \underline{w})\right]^{*} U_{\underline{a}^{\prime}, \underline{b}^{\prime}}^{(\mathrm{n})}(\mathrm{g}, \underline{\mathrm{h}}) \Omega\right\rangle \\
& =\left\langle\xi_{[0, t]}, \eta_{[0, t]}\right\rangle\left\langle\zeta_{[t, T]}, \gamma_{[t, T]}\right\rangle \text {. }
\end{aligned}
$$

Thus we get $\left\langle\Xi_{t}\left(\xi_{[0, t]} \otimes \zeta_{[t, T]}\right), \Xi_{t}\left(\eta_{[0, t]} \otimes \gamma_{[t, T]}\right)\right\rangle=\left\langle\xi_{[0, t]} \otimes \zeta_{[t, T]}, \eta_{[0, t]} \otimes \gamma_{[t, T]}\right\rangle$. Since by definition the range of $\Xi_{t}$ is dense in $\mathcal{H}_{T]}$, this proves $\Xi_{t}$ is a unitary operator.

Again by similar arguments to those above, for any $u, v \in \mathbf{h}$, we have

$$
\begin{aligned}
& \left\langle\Xi_{t} \xi_{[0, t]} \otimes \zeta_{[t, T]}, U_{t}(u, v) \Xi_{t} \eta_{[0, t]} \otimes \gamma_{[t, T]}\right\rangle \\
& =\left\langle U_{\underline{r}, \underline{s}}^{(n)}(\underline{u}, \underline{v}) \Omega, U_{t}(u, v) U_{\underline{a}, \underline{b}}^{(n)}(\underline{\mathrm{x}}, \underline{\mathrm{y}}) \Omega\right\rangle \\
& \left\langle U_{\underline{\underline{r}}^{\prime}, \underline{s}^{\prime}}^{(n)}(\underline{p}, \underline{w}) \Omega, U_{\underline{a}^{\prime}, \underline{b}^{\prime}}^{(n)}(\mathrm{g}, \underline{\mathrm{h}}) \Omega\right\rangle \\
& =\left\langle\xi_{[0, t]}, U_{t}(u, v) \eta_{[0, t]}\right\rangle\left\langle\zeta_{[t, T]}, \gamma_{[t, T]}\right\rangle .
\end{aligned}
$$

This proves (5.1).

\section{$\S 6 . \quad$ Expectation Semigroups}

Let us look at the various semigroups associated with the unitary evolution $\left\{U_{s, t}\right\}$. For any fixed $n \geq 1$, we define a family of operators $\left\{T_{t}^{(n)}\right\}$ on $\mathbf{h}^{\otimes n}$ by setting

$$
\left\langle\phi, T_{t}^{(n)} \psi\right\rangle:=\left\langle\Omega, U_{t}^{(n)}(\phi, \psi) \Omega\right\rangle, \forall \phi, \psi \in \mathbf{h}^{\otimes n} .
$$

Then in particular for product vectors $\underline{u}=\otimes_{i=1}^{n} u_{i}, \underline{v}=\otimes_{i=1}^{n} v_{i} \in \mathbf{h}^{\otimes n}$

$$
\left\langle\underline{u}, T_{t}^{(n)} \underline{v}\right\rangle=\left\langle\Omega, U_{t}^{(n)}(\underline{u}, \underline{v}) \Omega\right\rangle=\left\langle\Omega, U_{t}\left(u_{1}, v_{1}\right) U_{t}\left(u_{2}, v_{2}\right) \cdots U_{t}\left(u_{n}, v_{n}\right) \Omega\right\rangle .
$$

For $n=1$, we shall write $T_{t}$ for the family $T_{t}^{(1)}$.

Lemma 6.1. The above family of operators $\left\{T_{t}^{(n)}\right\}$ is a semigroup of contractions on $\mathbf{h}^{\otimes n}$. 
Proof. Since $U_{t}^{(n)}$ is in particular contractive, for any $\phi, \psi \in \mathbf{h}^{\otimes n}$

$$
\left|\left\langle\phi, T_{t}^{(n)} \psi\right\rangle\right|=\left|\left\langle\phi \Omega, U_{t}^{(n)} \psi \Omega\right\rangle\right| \leq\|\phi\|\|\psi\|
$$

and contractivity of $T_{t}^{(n)}$ follows.

In order to prove that this family of contractions $T_{t}^{(n)}$ is a semigroup it is enough to show that for any $0 \leq s \leq t$ and product vectors $\underline{u}=\otimes_{i=1}^{n} u_{i}, \underline{v}=$ $\otimes_{i=1}^{n} v_{i} \in \mathbf{h}^{\otimes n}$,

$$
\left\langle\underline{u}, T_{t}^{(n)} \underline{v}\right\rangle=\left\langle\underline{u}, T_{s}^{(n)} T_{t-s}^{(n)} \underline{v}\right\rangle
$$

Consider the product orthonormal basis $\left\{e_{j}=e_{j_{1}} \otimes e_{j_{2}} \otimes \cdots \otimes e_{j_{n}}: j=\right.$ $\left.\left(j_{1}, j_{2}, \cdots, j_{n}\right): j_{1}, j_{2}, \cdots, j_{n} \geq 1\right\}$ of $\mathbf{h}^{\otimes n}$. By part (iii) of Lemma 2.1 and the evolution property (4.5) of $U_{t}^{(n)}$,

$$
\begin{aligned}
& \left\langle\underline{u}, T_{t}^{(n)} \underline{v}\right\rangle=\left\langle\Omega, U_{t}^{(n)}(\underline{u}, \underline{v}) \Omega\right\rangle \\
& =\sum_{j}\left\langle\Omega, U_{s}^{(n)}\left(\underline{u}, e_{\underline{j}}\right) U_{s, t}^{(n)}\left(e_{\underline{j}}, \underline{v}\right) \Omega\right\rangle \\
& =\sum_{\dot{j}}\left\langle\Omega, U_{s}^{(n)}\left(\underline{u}, e_{\underline{j}}\right) \Omega\right\rangle\left\langle\Omega, U_{t-s}^{(n)}\left(e_{\underline{j}}, \underline{v}\right) \Omega\right\rangle \\
& =\sum_{\dot{j}}\left\langle\underline{u}, T_{s}^{(n)} e_{\underline{j}}\right\rangle\left\langle e_{\underline{i}}, T_{t-s}^{(n)} \underline{v}\right\rangle=\left\langle\underline{u}, T_{s}^{(n)} T_{t-s}^{(n)} \underline{v}\right\rangle .
\end{aligned}
$$

The following Lemma will be needed in the sequel

Lemma 6.2. (i) For $1 \leq k \leq n$,

$$
\left\langle\Omega, U_{t}^{(n, k)}(\underline{p}, \underline{w}) \Omega\right\rangle=\left\langle\underline{p}, T_{t}^{(n, k)} \underline{w}\right\rangle, \forall \underline{p}, \underline{w} \in \mathbf{h}^{\otimes n}
$$

where $T_{t}^{(n, k)}=1_{\mathbf{h}^{(\otimes k-1)}} \otimes T_{t} \otimes 1_{\mathbf{h}^{(\otimes n-k)}}$.

(ii) For any $1 \leq m \leq n, \underline{p}, \underline{w} \in \mathbf{h}^{\otimes n}$,

$$
\left\langle\Omega,\left(\prod_{k=1}^{m} U_{t}^{(n, k)}\right)(\underline{p}, \underline{w}) \Omega\right\rangle=\left\langle\underline{p}, T_{t}^{(m)} \otimes 1_{\mathbf{h}^{(\otimes n-m)}} \underline{w}\right\rangle .
$$

(iii) For any $\phi \in \mathbf{h}^{\otimes n}$,

$$
\begin{aligned}
& \left\|\left(U_{t}^{(n, k)}-1\right) \phi \otimes \Omega\right\|^{2} \\
& =\left\langle\left(1-T_{t}^{(n, k)}\right) \phi, \phi\right\rangle+\left\langle\phi,\left(1-T_{t}^{(n, k)}\right) \phi\right\rangle \\
& \leq 2\left\|1-T_{t}\right\|\|\phi\|^{2} .
\end{aligned}
$$


(iv)

$$
\begin{aligned}
& \left\|\left(U_{t}^{(n)}-1\right) \phi \otimes \Omega\right\|^{2} \\
& =\left\langle\left(1-T_{t}^{(n)}\right) \phi, \phi\right\rangle+\left\langle\phi,\left(1-T_{t}^{(n)}\right) \phi\right\rangle \\
& \leq 2\left\|\left(1-T_{t}^{(n)}\right)\right\|\|\phi\|^{2} .
\end{aligned}
$$

(v) For any $v \in \mathbf{h}$

$$
\sum_{m \geq 1}\left\|\left(U_{t}-1\right)\left(e_{m}, v\right) \Omega\right\|^{2}=2 \operatorname{Re}\left\langle v,\left(1-T_{t}\right) v\right\rangle \leq 2\|v\|^{2}\left\|T_{t}-1\right\| .
$$

Proof. (i) It follows from the fact that for product vectors $\underline{p}, \underline{w}$

$$
\left\langle\Omega, U_{t}^{(n, k)}(\underline{p}, \underline{w}) \Omega\right\rangle=\left\langle p_{k}, T_{t}^{(n, k)} w_{k}\right\rangle \prod_{i \neq k}\left\langle p_{i}, w_{i}\right\rangle .
$$

Part (ii) follows from Lemma 4.3 (ii).

The proofs of (iii) and (iv) are similar so we give the proof only for $U_{t}^{(n, k)}$. We have

$$
\begin{aligned}
& \left\|\left(U_{t}^{(n, k)}-1\right) \phi \Omega\right\|^{2} \\
& =\left\langle\phi \Omega,\left[\left(U_{t}^{(n, k)}-1\right)^{*}\left(U_{t}^{(n, k)}-1\right)\right] \phi \Omega\right\rangle \\
& =\left\langle\phi \Omega,\left[2-\left(U_{t}^{(n, k)}\right)^{*}-U_{t}^{(n, k)}\right] \phi \Omega\right\rangle \quad\left(\text { since } U_{t}^{(n, k)} \text { is an unitary operator }\right) \\
& =\left\langle\left(1-T_{t}^{(n, k)}\right) \phi, \phi\right\rangle+\left\langle\phi,\left(1-T_{t}^{(n, k)}\right) \phi\right\rangle
\end{aligned}
$$

Thus the statement follows.

(v) For any $v \in \mathbf{h}$

$$
\begin{aligned}
& \sum_{m \geq 1}\left\|\left(U_{t}-1\right)\left(e_{m}, v\right) \Omega\right\|^{2} \\
& \left.=\sum_{m \geq 1}\left\langle\Omega,\left(U_{t}-1\right)^{*}\left(v, e_{m}\right)\right)\left(U_{t}-1\right)\left(e_{m}, v\right) \Omega\right\rangle \\
& =\left\langle\Omega,\left[\left(U_{t}-1\right)^{*}\left(U_{t}-1\right)\right](v, v) \Omega\right\rangle \\
& =\left\langle\Omega,\left[2-U_{t}^{*}-U_{t}\right](v, v) \Omega\right\rangle \\
& =\left\langle v,\left[2-T_{t}^{*}-T_{t}\right] v\right\rangle=2 \operatorname{Re}\left\langle v,\left(1-T_{t}\right) v\right\rangle=2\|v\|^{2}\left\|T_{t}-1\right\| .
\end{aligned}
$$

Now we are ready to prove

Proposition 6.3. $\quad$ Under the Assumption B the semigroup $\left\{T_{t}^{(n)}\right\}$ is uniformly continuous. 
Proof. Assumption B on the family of unitary operators $\left\{U_{s, t}\right\}$ implies that the semigroup of contractions $\left\{T_{t}\right\}$ on $\mathbf{h}$ is uniformly continuous. To apply induction let us assume that for some $m \geq 1$, the contractive semigroups $\left\{T_{t}^{(n)}\right\}$ are uniformly continuous for all $1 \leq n \leq m-1$. Now, for any $\phi, \psi \in \mathbf{h}^{\otimes m}$

$$
\begin{aligned}
\left\langle\phi \otimes \Omega,\left(U_{t}^{(m)}-1\right) \psi \otimes \Omega\right\rangle \\
=\left\langle\phi \otimes \Omega,\left(\left[\prod_{k=1}^{m-1} U_{t}^{(m, k)}\right]\left[U_{t}^{(m, m)}\right]-1\right) \psi \otimes \Omega\right\rangle \\
=\left\langle\left[\prod_{k=1}^{m-1} U_{t}^{(m, k)}\right]^{*} \phi \otimes \Omega,\left(\left[U_{t}^{(m, m)}\right]-1\right) \psi \otimes \Omega\right\rangle \\
\quad+\left\langle\phi \otimes \Omega,\left(\left[\prod_{k=1}^{m-1} U_{t}^{(m, k)}\right]-1\right) \psi \otimes \Omega\right\rangle .
\end{aligned}
$$

Taking absolute values, by Lemma 6.2 we get

$$
\begin{aligned}
& \left|\left\langle\phi,\left(T_{t}^{(m)}-1_{\mathbf{h} \otimes m}\right) \psi\right\rangle\right| \\
& \leq\|\phi\|\|\psi\| \sqrt{2\left\|T_{t}^{(m, m)}-1_{\mathbf{h} \otimes m}\right\|}+\left|\left\langle\phi,\left(\left[T_{t}^{(m-1)} \otimes 1_{\mathbf{h}}\right]-1_{\mathbf{h} \otimes m}\right) \psi\right\rangle\right| \\
& \leq\|\phi\|\|\psi\|\left[\sqrt{2\left\|T_{t}-1\right\|}+\left\|T_{t}^{(m-1)}-1\right\|\right] .
\end{aligned}
$$

So uniform continuity of $T_{t}^{(m-1)}$ and $T_{t}$ implies that $T_{t}^{(m)}$ is uniformly continuous.

Let us denote the bounded generator of the uniformly continuous semigroup $T_{t}^{(n)}$ on $\mathbf{h}^{\otimes n}$ by $G^{(n)}$ and for $n=1$ by $G$.

For $n \geq 1$, we define a family of operators $\left\{Z_{t}^{(n)}: t \geq 0\right\}$ on the Banach space $\mathcal{B}_{1}\left(\mathbf{h}^{\otimes n}\right)$ by

$$
Z_{t}^{(n)} \rho=\operatorname{Tr}_{\mathcal{H}}\left[U_{t}^{(n)}(\rho \otimes|\Omega><\Omega|)\left(U_{t}^{(n)}\right)^{*}\right], \rho \in \mathcal{B}_{1}\left(\mathbf{h}^{\mathbf{h}^{\otimes n}}\right) .
$$

Then in particular for product vectors $\underline{u}, \underline{v}, \underline{p}, \underline{w} \in \mathbf{h}^{\otimes n}$.

$$
\left\langle\underline{p}, Z_{t}^{(n)}(|\underline{w}\rangle\langle\underline{v}|) \underline{u}\right\rangle:=\left\langle U_{t}^{(n)}(\underline{u}, \underline{v}) \Omega, U_{t}^{(n)}(\underline{p}, \underline{w}) \Omega\right\rangle .
$$

Lemma 6.4. The above family $\left\{Z_{t}^{(n)}\right\}$ is a semigroup of contractive maps on $\mathcal{B}_{1}\left(\mathbf{h}^{\otimes n}\right)$. Furthermore, Assumption $\mathbf{B}$ implies that $\left\{Z_{t}^{(n)}\right\}$ is uniformly continuous. 
Proof. For $\rho \in \mathcal{B}_{1}\left(\mathbf{h}^{\otimes n}\right)$

$$
\begin{aligned}
& \left\|Z_{t}^{(n)} \rho\right\|_{1}=\left\|\operatorname{Tr}_{\mathcal{H}}\left[U_{t}^{(n)}(\rho \otimes|\Omega><\Omega|)\left(U_{t}^{(n)}\right)^{*}\right]\right\|_{1} \\
& =\sup _{\phi^{(n)}}\left\{\sum_{k \geq 1}\left|\left\langle\phi_{k}^{(n)}, \operatorname{Tr}_{\mathcal{H}}\left[U_{t}^{(n)}(\rho \otimes|\Omega><\Omega|)\left(U_{t}^{(n)}\right)^{*}\right] \phi_{k}^{(n)}\right\rangle\right|,\right.
\end{aligned}
$$

(where the supremum is over all ONB $\phi^{(n)} \equiv\left\{\phi_{k}^{(n)}\right\}$ of $\mathbf{h}^{\otimes n}$ )

$$
\begin{aligned}
& \leq \sup _{\phi^{(n)}} \sum_{j, k \geq 1}\left|\left\langle\phi_{k}^{(n)} \otimes \zeta_{j}, U_{t}^{(n)}(\rho \otimes|\Omega><\Omega|)\left(U_{t}^{(n)}\right)^{*} \phi_{k}^{(n)} \otimes \zeta_{j}\right\rangle\right| \\
& \leq\left\|U_{t}^{(n)}(\rho \otimes|\Omega><\Omega|)\left(U_{t}^{(n)}\right)^{*}\right\|_{1} .
\end{aligned}
$$

Since $\left\{U_{t}^{(n)}\right\}$ is in particular a contractive family of operators

$$
\left\|Z_{t}^{(n)} \rho\right\|_{1} \leq\|\rho \otimes|\Omega><\Omega|\|_{1}=\|\rho\|_{1} .
$$

In order to prove that the family of contractions $\left\{Z_{t}^{(n)}\right\}$ is a semigroup it is enough to verify that property for the rank one operator $\rho=|\underline{w}><\underline{v}|: \underline{v}=$ $\otimes_{i=1}^{n} v_{i}, \underline{w}=\otimes_{i=1}^{n} w_{i} \in \mathbf{h}^{\otimes n}$. Therefore, it suffices to prove that

$$
\left\langle\underline{p}, Z_{t}^{(n)}(\rho) \underline{u}\right\rangle=\left\langle\underline{p}, Z_{s}^{(n)} Z_{t-s}^{(n)}(\rho) \underline{u}\right\rangle, \forall \underline{p}=\otimes_{i=1}^{n} p_{i}, \underline{u}=\otimes_{i=1}^{n} u_{i} \in \mathbf{h}^{\otimes n} .
$$

By Lemma 4.3, part (iv) of Lemma 2.1 and Assumption A, for $0 \leq s \leq$ $t, \underline{u}, \underline{v} \in \mathbf{h}^{\otimes n}$ and product $\mathrm{ONB}\left\{e_{\underline{k}}^{(n)}=e_{k_{1}} \otimes \cdots \otimes e_{k_{n}}\right\}$ of $\mathbf{h}^{\otimes n}$

$$
\begin{aligned}
& \left\langle U_{t}^{(n)}(\underline{u}, \underline{v}) \Omega, U_{t}^{(n)}(\underline{p}, \underline{w}) \Omega\right\rangle \\
& =\sum_{i, \underline{k}}\left\langle U_{s}^{(n)}\left(\underline{u}, e_{\underline{i}}^{(n)}\right) \Omega, U_{s}^{(n)}\left(\underline{p}, e_{\underline{k}}^{(n)}\right) \Omega\right\rangle\left\langle U_{t-s}^{(n)}\left(e_{\underline{j}}^{(n)}, \underline{v}\right) \Omega, U_{t-s}^{(n)}\left(e_{\underline{k}}^{(n)}, \underline{p}\right) \Omega\right\rangle .
\end{aligned}
$$

This gives

$$
\begin{aligned}
& \left\langle\underline{p}, Z_{t}^{(n)}(\rho) \underline{u}\right\rangle \\
& =\sum_{j, \underline{k}}\left\langle\underline{p}, Z_{s}^{(n)}\left(\left|e_{\underline{k}}^{(n)}><e_{\underline{j}}^{(n)}\right|\right) \underline{u}\right\rangle\left\langle e_{\underline{k}}^{(n)}, Z_{t-s}^{(n)}(\rho) e_{\underline{j}}^{(n)}\right\rangle \\
& =\sum_{j, \underline{k}}\left\langle e_{\underline{i}}^{(n)},\left(Z_{s}^{(n)}\right)^{*}(|\underline{u}><\underline{p}|) e_{\underline{k}}^{(n)}\right\rangle\left\langle e_{\underline{k}}^{(n)}, Z_{t-s}^{(n)}(\rho) e_{\underline{i}}^{(n)}\right\rangle \\
& =\sum_{j}\left\langle e_{\dot{j}}^{(n)},\left(Z_{s}^{(n)}\right)^{*}(|\underline{u}><\underline{p}|) Z_{t-s}^{(n)}(\rho) e_{\dot{i}}^{(n)}\right\rangle \\
& =\operatorname{Tr}\left[\left(Z_{s}^{(n)}\right)^{*}(|\underline{u}><\underline{p}|) Z_{t-s}^{(n)}(\rho)\right] \\
& =\operatorname{Tr}\left[|\underline{u}><\underline{p}| Z_{s}^{(n)} Z_{t-s}^{(n)}(\rho)\right] \\
& =\left\langle\underline{p}, Z_{s}^{(n)} Z_{t-s}^{(n)}(\rho) \underline{u}\right\rangle \text {. }
\end{aligned}
$$


In order to prove uniform continuity of $Z_{t}^{(n)}$ we consider

$$
\begin{aligned}
& \left\|\left(Z_{t}^{(n)}-1\right)(|\underline{w}><\underline{v}|)\right\|_{1} \\
& =\sup _{\phi^{(n)}}\left\{\sum_{k \geq 1}\left|\left\langle\phi_{k}^{(n)},\left(Z_{t}^{(n)}-1\right)(|\underline{w}><\underline{v}|) \phi_{k}^{(n)}\right\rangle\right|\right\} \\
& =\sup _{\phi^{(n)}} \sum_{k \geq 1}\left|\left\langle U_{t}^{(n)}\left(\phi_{k}^{(n)}, \underline{v}\right) \Omega, U_{t}^{(n)}\left(\phi_{k}^{(n)}, \underline{w}\right) \Omega\right\rangle-\overline{\left\langle\phi_{k}^{(n)}, \underline{v}\right\rangle}\left\langle\phi_{k}^{(n)}, \underline{w}\right\rangle\right| \\
& \leq \sup _{\phi^{(n)}} \sum_{k \geq 1}\left|\left\langle\left(U_{t}^{(n)}-1\right)\left(\phi_{k}^{(n)}, \underline{v}\right) \Omega, U_{t}^{(n)}\left(\phi_{k}^{(n)}, \underline{w}\right) \Omega\right\rangle\right| \\
& +\sup _{\phi^{(n)}} \sum_{k \geq 1} \overline{\mid\left\langle\phi_{k}^{(n)}, \underline{v}\right\rangle}\left\langle\Omega,\left(U_{t}^{(n)}-1\right)\left(\phi_{k}^{(n)}, \underline{w}\right) \Omega\right\rangle \mid \\
& \leq \sup _{\phi^{(n)}}\left[\sum_{k \geq 1}\left\|\left(U_{t}^{(n)}-1\right)\left(\phi_{k}^{(n)}, \underline{v}\right) \Omega\right\|^{2}\right]^{\frac{1}{2}}\left[\sum_{k \geq 1}\left\|U_{t}^{(n)}\left(\phi_{k}^{(n)}, \underline{w}\right) \Omega\right\|^{2}\right]^{\frac{1}{2}} \\
& +\sup _{\phi^{(n)}}\left[\sum_{k \geq 1} \mid\left\langle\phi_{k}^{(n)},\left.\underline{v}\right|^{2}\right]^{\frac{1}{2}}\left[\sum_{k \geq 1}\left\|\left(U_{t}^{(n)}-1\right)\left(\phi_{k}^{(n)}, \underline{w}\right) \Omega\right\|^{2}\right]^{\frac{1}{2}}\right.
\end{aligned}
$$

So by Lemma 6.2

$$
\begin{aligned}
& \left\|\left(Z_{t}^{(n)}-1\right)(|\underline{w}><\underline{v}|)\right\|_{1} \\
& \leq 2 \sqrt{2}\|\underline{v}\|\|\underline{w}\|\left(\sqrt{\left\|T_{t}^{(n)}-1\right\|}\right) .
\end{aligned}
$$

Now for any $\rho=\sum_{k} \lambda_{k}\left|\phi_{k}^{(n)}><\phi_{k}^{(n)}\right| \in \mathcal{B}_{1}\left(\mathbf{h}^{\otimes n}\right)$ we have

$$
\begin{aligned}
& \left\|Z_{t}^{(n)}(\rho)-\rho\right\|_{1} \\
& \leq 2 \sqrt{2} \sum_{k}\left|\lambda_{k}\right|\left(\sqrt{\left\|T_{t}^{(n)}-1\right\|}\right) \\
& \leq 2 \sqrt{2}\|\rho\|_{1}\left(\sqrt{\left\|T_{t}^{(n)}-1\right\|}\right) .
\end{aligned}
$$

Thus by uniform continuity of the semigroup $T_{t}^{(n)}$ it follows that the semigroup $Z_{t}^{(n)}$ is uniformly continuous on $\mathcal{B}_{1}\left(\mathbf{h}^{\otimes n}\right)$.

We shall denote the bounded generator of the semi-group $Z_{t}^{(n)}$ by $\mathcal{L}^{(n)}$ and for $n=1$ we simply write $Z_{t}$ and $\mathcal{L}$ for the semigroup $Z_{t}^{(1)}$ and its generator $\mathcal{L}^{(1)}$ respectively. 
Lemma 6.5. For any $n \geq 1, Z_{t}^{(n)}$ is a positive trace preserving semigroup on $\mathcal{B}_{1}\left(\mathbf{h}^{\otimes n}\right)$.

Proof. Positivity follows from

$$
\left\langle\underline{u}, Z_{t}^{(n)}(|\underline{v}><\underline{v}|) \underline{u}\right\rangle=\left\|U_{t}^{(n)}(\underline{u}, \underline{v}) \Omega\right\|^{2} \geq 0 \forall \underline{u}, \underline{v} \in \mathbf{h}^{\otimes n} .
$$

To prove that $Z_{t}^{(n)}$ is trace preserving it is enough to show that

$$
\operatorname{Tr}\left[Z_{t}^{(n)}(|\underline{u}><\underline{v}|)\right]=\langle\underline{v}, \underline{u}\rangle .
$$

By definition and Lemma 2.1

$$
\begin{aligned}
& \operatorname{Tr}\left[Z_{t}^{(n)}(|\underline{u}><\underline{v}|)\right]=\sum_{k}\left\langle\underline{\mathrm{e}}_{k}, Z_{t}^{(n)}(|\underline{u}><\underline{v}|) \underline{\mathrm{e}}_{k}\right\rangle \\
& =\sum_{k}\left\langle U_{t}^{(n)}\left(\underline{\mathrm{e}}_{k}, \underline{v}\right) \Omega, U_{t}^{(n)}\left(\underline{\mathrm{e}}_{k}, \underline{u}\right) \Omega\right\rangle \\
& =\left\langle\Omega,\left(U_{t}^{(n)}\right)^{*} U_{t}^{(n)}(\underline{v}, \underline{u}) \Omega\right\rangle .
\end{aligned}
$$

Since $U_{t}^{(n)}$ is unitary, we get

$$
\operatorname{Tr}\left[Z_{t}^{(n)}(|\underline{u}><\underline{v}|)\right]=\langle\underline{v}, \underline{u}\rangle .
$$

The above Lemma yields

$$
\operatorname{Tr}\left(\mathcal{L}^{(n)} \rho\right)=0, \forall \rho \in \mathcal{B}_{1}\left(\mathbf{h}^{\otimes n}\right) .
$$

We conclude this section by the following useful observations.

Lemma 6.6. Under the Assumption C, for any $n \geq 3, \underline{u}, \underline{v} \in \mathbf{h}^{\otimes n}$, $\underline{\epsilon} \in \mathbb{Z}_{2}^{n}$

(6.6) $\quad \lim _{t \rightarrow 0} \frac{1}{t}\left\langle\Omega,\left(U_{t}^{\left(\epsilon_{1}\right)}-1\right)\left(u_{1}, v_{1}\right) \cdots\left(U_{t}^{\left(\epsilon_{n}\right)}-1\right)\left(u_{n}, v_{n}\right) \Omega\right\rangle=0$.

Proof. We have

$$
\begin{aligned}
& \mid \frac{1}{t}\left\langle\left[\left(U_{t}^{\left(\epsilon_{1}\right)}-1\right)\left(u_{1}, v_{1}\right)\left(U_{t}^{\left(\epsilon_{2}\right)}-1\right)\left(u_{2}, v_{2}\right)\right]^{*} \Omega\right. \\
& \left.\left(U_{t}^{\left(\epsilon_{1}\right)}-3\right)\left(u_{3}, v_{3}\right) \cdots\left(U_{t}^{\left(\epsilon_{n}\right)}-1\right)\left(u_{n}, v_{n}\right) \Omega\right\rangle\left.\right|^{2} \\
& \leq \frac{1}{t^{2}}\left\|\left[\left(U_{t}^{\left(\epsilon_{1}\right)}-1\right)\left(u_{1}, v_{1}\right)\left(U_{t}^{\left(\epsilon_{2}\right)}-1\right)\left(u_{2}, v_{2}\right)\right]^{*} \Omega\right\|^{2} \\
& \left\|\left(U_{t}^{\epsilon_{3}}-1\right)\left(u_{3}, v_{3}\right) \cdots\left(U_{t}^{\left(\epsilon_{n}\right)}-1\right)\left(u_{n}, v_{n}\right) \Omega\right\|^{2} \\
& \leq C_{\underline{u}, \underline{v}} \frac{1}{t}\left\|\left[\left(U_{t}^{\left(\epsilon_{1}\right)}-1\right)\left(u_{1}, v_{1}\right)\left(U_{t}^{\left(\epsilon_{2}\right)}-1\right)\left(u_{2}, v_{2}\right)\right]^{*} \Omega\right\|^{2} \\
& \frac{1}{t}\left\|\left(U_{t}^{\left(\epsilon_{n-1}\right)}-1\right)\left(u_{n-1}, v_{n-1}\right)\left(U_{t}^{\left(\epsilon_{n}\right)}-1\right)\left(u_{n}, v_{n}\right) \Omega\right\|^{2}
\end{aligned}
$$


for some constant $C_{\underline{u}, \underline{v}}$ independent of $t$. So to prove (6.6) it is enough to show that for any $u, v, p, w \in \mathbf{h}$ and $\epsilon, \epsilon^{\prime} \in \mathbb{Z}_{2}$

$$
\lim _{t \rightarrow 0} \frac{1}{t}\left\|\left(U_{t}^{(\epsilon)}-1\right)(u, v)\left(U_{t}^{\left(\epsilon^{\prime}\right)}-1\right)(p, w) \Omega\right\|^{2}=0 .
$$

So let us look at the following

$$
\begin{aligned}
& \left\|\left(U_{t}^{(\epsilon)}-1\right)(u, v)\left(U_{t}^{\left(\epsilon^{\prime}\right)}-1\right)(p, w) \Omega\right\|^{2} \\
& =\left\langle\left(U_{t}^{(\epsilon)}-1\right)(u, v)\left(U_{t}^{\left(\epsilon^{\prime}\right)}-1\right)(p, w) \Omega,\left(U_{t}^{(\epsilon)}-1\right)(u, v)\left(U_{t}^{\left(\epsilon^{\prime}\right)}-1\right)(p, w) \Omega\right\rangle \\
& =\left\langle\left(U_{t}^{\left(\epsilon^{\prime}\right)}-1\right)(p, w) \Omega,\left[\left(U_{t}^{(\epsilon)}-1\right)(u, v)\right]^{*}\left(U_{t}^{(\epsilon)}-1\right)(u, v)\left(U_{t}^{\left(\epsilon^{\prime}\right)}-1\right)(p, w) \Omega\right\rangle .
\end{aligned}
$$

By part (v) of Lemma 2.1 the above quantity is

$\leq\|u\|^{2}\left\langle\left(U_{t}^{\left(\epsilon^{\prime}\right)}-1\right)(p, w) \Omega,\left[\left(U_{t}^{(\epsilon)}-1\right)^{*}\left(U_{t}^{(\epsilon)}-1\right)\right](v, v)\left(U_{t}^{\left(\epsilon^{\prime}\right)}-1\right)(p, w) \Omega\right\rangle$. Since by contractivity of the family $U_{t}^{(\epsilon)},\left(U_{t}^{(\epsilon)}\right)^{*} U_{t}^{(\epsilon)} \leq 1$, we get

$$
\begin{aligned}
& \left\|\left(U_{t}^{(\epsilon)}-1\right)(u, v)\left(U_{t}^{\left(\epsilon^{\prime}\right)}-1\right)(p, w) \Omega\right\|^{2} \\
& \leq\|u\|^{2}\left\langle\left(U_{t}^{\left(\epsilon^{\prime}\right)}-1\right)(p, w) \Omega,\left[1-\left(U_{t}^{(\epsilon)}\right)^{*}+1-U_{t}^{(\epsilon)}\right](v, v)\left(U_{t}^{\left(\epsilon^{\prime}\right)}-1\right)(p, w) \Omega\right\rangle \\
& =-\|u\|^{2}\left\langle\left(U_{t}^{\left(\epsilon^{\prime}\right)}-1\right)(p, w) \Omega,\left[U_{t}^{(1+\epsilon)}-1\right](v, v)\left(U_{t}^{\left(\epsilon^{\prime}\right)}-1\right)(p, w) \Omega\right\rangle \\
& \quad-\|u\|^{2}\left\langle\left(U_{t}^{\left(\epsilon^{\prime}\right)}-1\right)(p, w) \Omega,\left(U_{t}^{(\epsilon)}-1\right)(v, v)\left(U_{t}^{\left(\epsilon^{\prime}\right)}-1\right)(p, w) \Omega\right\rangle .
\end{aligned}
$$

Thus by Assumption $\mathbf{C}$ we get (6.7) and the proof is complete.

Lemma 6.7. For vectors $u, v \in \mathbf{h}$, product vectors $\underline{p}, \underline{w} \in \mathbf{h}^{\otimes n}$ and $\epsilon \in$ $\mathbb{Z}_{2}, \underline{\epsilon^{\prime}} \in \mathbb{Z}_{2}^{n}$

$$
\begin{gathered}
\lim _{t \rightarrow 0} \frac{1}{t}\left\langle\left(U_{t}-1\right)^{(\epsilon)}(u, v) \Omega,\left(U_{t}^{\left(\underline{\epsilon}^{\prime}\right)}-1\right)(\underline{p}, \underline{w}) \Omega\right\rangle \\
=(-1)^{\epsilon} \lim _{t \rightarrow 0} \frac{1}{t}\left\langle\left(U_{t}-1\right)(u, v) \Omega,\left(U_{t}^{\left(\underline{\epsilon}^{\prime}\right)}-1\right)(\underline{p}, \underline{w}) \Omega\right\rangle .
\end{gathered}
$$

Proof. For $\epsilon=0$ there is nothing to prove. To see this for $\epsilon=1$ consider the following

$$
\text { (6.9) } \begin{aligned}
& \lim _{t \rightarrow 0} \frac{1}{t}\left\langle\left(U_{t}+U_{t}^{*}-2\right)(u, v) \Omega,\left(U_{t}^{\left(\underline{\epsilon}^{\prime}\right)}-1\right)(\underline{p}, \underline{w}) \Omega\right\rangle \\
& =-\lim _{t \rightarrow 0} \frac{1}{t}\left\langle\left[\left(U_{t}^{*}-1\right)\left(U_{t}-1\right)\right](u, v) \Omega,\left(U_{t}^{\left(\underline{\epsilon}^{\prime}\right)}-1\right)(\underline{p}, \underline{w}) \Omega\right\rangle \\
& =-\lim _{t \rightarrow 0} \frac{1}{t} \sum_{m \geq 1}\left\langle\left(U_{t}-1\right)\left(e_{m}, v\right) \Omega,\left(U_{t}-1\right)\left(e_{m}, u\right)\left(U_{t}^{\left(\underline{\epsilon}^{\prime}\right)}-1\right)(\underline{p}, \underline{w}) \Omega\right\rangle .
\end{aligned}
$$


That this limit vanishes can be seen from the following

$$
\begin{aligned}
& \left|\frac{1}{t} \sum_{m \geq 1}\left\langle\left(U_{t}-1\right)\left(e_{m}, v\right) \Omega,\left(U_{t}-1\right)\left(e_{m}, u\right)\left(U_{t}^{(\underline{\epsilon})}-1\right)(\underline{p}, \underline{w}) \Omega\right\rangle\right|^{2} \\
& \leq \sum_{m \geq 1} \frac{1}{t}\left\|\left(U_{t}-1\right)\left(e_{m}, v\right) \Omega\right\|^{2} \sum_{m \geq 1} \frac{1}{t}\left\|\left(U_{t}-1\right)\left(e_{m}, u\right)\left(U_{t}^{(\underline{\epsilon})}-1\right)(\underline{p}, \underline{w}) \Omega\right\|^{2} .
\end{aligned}
$$

By Lemma $6.2(\mathrm{v})$ and Lemma 2.1 (iv) the above quantity is equal to

$$
\begin{aligned}
& 2 \operatorname{Re}\left\langle v, \frac{1-T_{t}}{t} v\right\rangle \frac{1}{t}\left\langle\left(U_{t}^{(\underline{\epsilon})}-1\right)(\underline{p}, \underline{w}) \Omega,\left[\left(U_{t}^{*}-1\right)\left(U_{t}-1\right)\right](u, u)\left(U_{t}^{(\underline{\epsilon})}-1\right)(\underline{p}, \underline{w}) \Omega\right\rangle \\
& \leq 2 \operatorname{Re}\left\langle v, \frac{1-T_{t}}{t} v\right\rangle \frac{1}{t}\left\langle\left(U_{t}^{(\underline{\epsilon})}-1\right)(\underline{p}, \underline{w}) \Omega,\left(2-U_{t}^{*}-U_{t}\right)(u, u)\left(U_{t}^{(\underline{\epsilon})}-1\right)(\underline{p}, \underline{w}) \Omega\right\rangle
\end{aligned}
$$

Therefore, since $T_{t}$ is continuous, by Assumption $\mathbf{C}$

$$
\lim _{t \rightarrow 0} \frac{1}{t} \sum_{m \geq 1}\left\langle\left(U_{t}-1\right)\left(e_{m}, v\right) \Omega,\left(U_{t}-1\right)\left(e_{m}, u\right)\left(U_{t}^{(\underline{\epsilon})}-1\right)(\underline{p}, \underline{w}) \Omega\right\rangle=0 .
$$

In particular for vectors $u, v, p, w \in \mathbf{h}$ the identity (6.8) gives

$$
\begin{gathered}
\lim _{t \rightarrow 0} \frac{1}{t}\left\langle\left(U_{t}-1\right)^{(\epsilon)}(u, v) \Omega,\left(U_{t}-1\right)^{\epsilon^{\prime}}(p, w) \Omega\right\rangle \\
=(-1)^{\epsilon+\epsilon^{\prime}} \lim _{t \rightarrow 0} \frac{1}{t}\left\langle\left(U_{t}-1\right)(u, v) \Omega,\left(U_{t}-1\right)(p, w) \Omega\right\rangle .
\end{gathered}
$$

\section{$\S 7 . \quad$ Representation of Hilbert Tensor Algebra and Construction of} Noise Space

We define a scalar valued map $K$ on $M \times M$ by setting, for $(\underline{u}, \underline{v}, \underline{\epsilon}),\left(\underline{p}, \underline{w}, \underline{\epsilon}^{\prime}\right)$ $\in M_{0}$,

$K\left((\underline{u}, \underline{v}, \underline{\epsilon}),\left(\underline{p}, \underline{w}, \underline{\epsilon}^{\prime}\right)\right):=\lim _{t \rightarrow 0} \frac{1}{t}\left\langle\left(U_{t}^{(\underline{\epsilon})}-1\right)(\underline{u}, \underline{v}) \Omega,\left(U_{t}^{\epsilon^{\prime}}-1\right)(\underline{p}, \underline{w}) \Omega\right\rangle$, when it exists.

Lemma 7.1. (i) The map $K$ is a well defined positive definite kernel on $M$.

(ii) Up to unitary equivalence there exists a unique separable Hilbert space $\mathbf{k}$, an embedding $\eta: M \rightarrow \mathbf{k}$ and a *-representation $\pi$ of $M, \pi: M \rightarrow \mathcal{B}(\mathbf{k})$ such that

$$
\left\{\eta(\underline{u}, \underline{v}, \underline{\epsilon}):(\underline{u}, \underline{v}, \underline{\epsilon}) \in M_{0}\right\} \text { is total in } \mathbf{k} \text {, }
$$




$$
\left\langle\eta(\underline{u}, \underline{v}, \underline{\epsilon}), \eta\left(\underline{p}, \underline{w}, \underline{\epsilon}^{\prime}\right)\right\rangle=K\left((\underline{u}, \underline{v}, \underline{\epsilon}),\left(\underline{p}, \underline{w}, \underline{\epsilon}^{\prime}\right)\right)
$$

and

$(7.3) \quad \pi(\underline{u}, \underline{v}, \underline{\epsilon}) \eta\left(\underline{p}, \underline{w}, \underline{\epsilon}^{\prime}\right)=\eta\left(\underline{u} \otimes \underline{p}, \underline{v} \otimes \underline{w}, \underline{\epsilon} \oplus \underline{\epsilon}^{\prime}\right)-\langle\underline{p}, \underline{w}\rangle \eta(\underline{u}, \underline{v}, \underline{\epsilon})$.

Proof. (i) First note that for any $(\underline{u}, \underline{v}, \underline{\epsilon}) \in M_{0}, \underline{u}=\otimes_{i=1}^{n} u_{i}, \underline{v}=\otimes_{i=1}^{n} v_{i}$, $\underline{\epsilon}=\left(\epsilon_{1}, \epsilon_{2}, \cdots, \epsilon_{n}\right)$ we can write

$$
\begin{aligned}
& \left(U_{t}^{(\underline{\epsilon})}-1\right)(\underline{u}, \underline{v})=\prod_{i=1}^{n} U_{t}^{\left(\epsilon_{i}\right)}\left(u_{i}, v_{i}\right)-\prod_{i=1}^{n}\left\langle u_{i}, v_{i}\right\rangle \\
& =\sum_{1 \leq i \leq n}\left(U_{t}-1\right)^{\left(\epsilon_{i}\right)}\left(u_{i}, v_{i}\right) \prod_{j \neq i}\left\langle u_{j}, v_{j}\right\rangle \\
& +\sum_{2 \leq l \leq n} \sum_{1 \leq i_{1}<\ldots<i_{m} \leq n} \prod_{k=1}^{l}\left(U_{t}-1\right)^{\epsilon_{i_{k}}}\left(u_{i_{k}}, v_{i_{k}}\right) \prod_{j \neq i_{k}}\left\langle u_{j}, v_{j}\right\rangle .
\end{aligned}
$$

Now by Lemma 6.6 , for elements $(\underline{u}, \underline{v}, \underline{\epsilon}),\left(\underline{p}, \underline{w}, \underline{\epsilon}^{\prime}\right) \in M_{0}, \underline{\epsilon} \in \mathbb{Z}_{2}^{m}$ and $\underline{\epsilon}^{\prime} \in \mathbb{Z}_{2}^{n}$, we have

$$
\begin{aligned}
& K\left((\underline{u}, \underline{v}, \underline{\epsilon}),\left(\underline{p}, \underline{w}, \underline{\epsilon}^{\prime}\right)\right)=\lim _{t \rightarrow 0} \frac{1}{t}\left\langle\left(U_{t}^{(\underline{\epsilon})}-1\right)(\underline{u}, \underline{v}) \Omega,\left(U_{t}^{\epsilon^{\prime}}-1\right)(\underline{p}, \underline{w}) \Omega\right\rangle \\
= & \sum_{1 \leq i \leq m, 1 \leq j \leq n}\left(\prod_{k \neq i} \overline{\left\langle u_{k}, v_{k}\right\rangle} \prod_{l \neq j}\left\langle p_{l}, w_{l}\right\rangle\right. \\
& \left.\times \lim _{t \rightarrow 0} \frac{1}{t}\left\langle\left(U_{t}-1\right)^{\left(\epsilon_{i}\right)}\left(u_{i}, v_{i}\right) \Omega,\left(U_{t}-1\right)^{\epsilon_{j}^{\prime}}\left(p_{j}, w_{j}\right) \Omega\right\rangle\right) .
\end{aligned}
$$

Hence by $(6.10) K\left((\underline{u}, \underline{v}, \underline{\epsilon}),\left(\underline{p}, \underline{w}, \underline{\epsilon}^{\prime}\right)\right)$ is given by

$$
\begin{aligned}
\sum_{1 \leq i \leq m, 1 \leq j \leq n} & (-1)^{\epsilon_{i}+\epsilon_{j}^{\prime}}\left(\prod_{k \neq i} \overline{\left\langle u_{k}, v_{k}\right\rangle} \prod_{l \neq j}\left\langle p_{l}, w_{l}\right\rangle\right. \\
& \left.\times \lim _{t \rightarrow 0} \frac{1}{t}\left\langle\left(U_{t}-1\right)\left(u_{i}, v_{i}\right) \Omega,\left(U_{t}-1\right)\left(p_{j}, w_{j}\right) \Omega\right\rangle\right) .
\end{aligned}
$$

Since $T_{t}$ and $Z_{t}$ are uniformly continuous semigroup existence of the above limit follows from the following

$$
\begin{aligned}
& \lim _{t \rightarrow 0} \frac{1}{t}\left\langle\left(U_{t}-1\right)(u, v) \Omega,\left(U_{t}-1\right)(p, w) \Omega\right\rangle \\
& =\lim _{t \rightarrow 0} \frac{1}{t}\left\{\left\langle U_{t}(u, v) \Omega, U_{t}(p, w) \Omega\right\rangle-\overline{\langle u, v\rangle}\langle p, w\rangle\right\} \\
& -\lim _{t \rightarrow 0} \frac{1}{t} \overline{\langle u, v\rangle}\left\langle\Omega,\left[\left(U_{t}-1\right)(p, w)\right] \Omega\right\rangle
\end{aligned}
$$




$$
\begin{aligned}
& -\lim _{t \rightarrow 0} \frac{1}{t} \overline{\left\langle\Omega,\left[\left(U_{t}-1\right)(u, v)\right] \Omega\right\rangle}\langle p, w\rangle \\
& =\lim _{t \rightarrow 0}\left\{\left\langle p, \frac{Z_{t}-1}{t}(|w\rangle\langle v|) u\right\rangle-\overline{\langle u, v\rangle}\left\langle p, \frac{T_{t}-1}{t} w\right\rangle-\overline{\left\langle u, \frac{T_{t}-1}{t} v\right\rangle}\langle p, w\rangle\right\}
\end{aligned}
$$

Thus $K$ is well defined on $M_{0}$. Now extend this to the $*$-algebra $M$ sesquilinearly.

In particular we have

$$
\begin{aligned}
K((u, v, 0),(p, w, 0))= & \langle p, \mathcal{L}(|w\rangle\langle v|) u\rangle-\overline{\langle u, v\rangle}\langle p, G w\rangle \\
& -\overline{\langle u, G v\rangle\langle p, w\rangle}
\end{aligned}
$$

where $\mathcal{L}$ and $G$ are generators of $Z_{t}$ and $T_{t}$ respectively. Positive definiteness of $K$ follows from the fact that, defining $\xi_{i}(t)=\left[U_{t}^{\left(\epsilon_{i}\right)}\left(\underline{u}_{i}, \underline{v}_{i}\right)-\left\langle\underline{u}_{i}, \underline{v}_{i}\right\rangle\right] \Omega$,

$$
\sum_{i, j=1}^{N} \bar{c}_{i} c_{j} K\left(\left(\underline{u}_{i}, \underline{v}_{i}, \underline{\epsilon}_{i}\right),\left(\underline{u}_{j}, \underline{v}_{j}, \underline{\epsilon}_{j}\right)\right)=\lim _{t \rightarrow 0} \frac{1}{t}\left\|\sum_{i=1}^{N} c_{i} \xi_{i}(t)\right\|^{2} \geq 0 .
$$

(ii) Kolmogorov's construction (Ref. [16]) to the pair $(M, K)$ gives the Hilbert space $\mathbf{k}$ and embedding $\eta$ satisfying (7.1). The separability of $\mathbf{k}$ follows from (7.1) and the verifiable fact $\|\eta(\underline{u}, \underline{v}, \underline{\epsilon})-\eta(\underline{p}, \underline{w}, \underline{\epsilon})\|_{\mathbf{k}} \rightarrow 0$ as $\|\underline{u}-\underline{p}\|$ and $\|\underline{v}-\underline{w}\| \rightarrow$ 0 .

Defining $\pi$ by $(7.3)$ we show that the map $\pi(\underline{u}, \underline{v}, \underline{\epsilon})$ extends to a bounded linear operator on $\mathbf{k}$ with $\|\pi(\underline{u}, \underline{v}, \underline{\epsilon})\| \leq\|\underline{u}\|\|\underline{v}\|$. For any $\xi=\sum_{i=1}^{N} c_{i} \eta\left(\underline{u}_{i}, \underline{v}_{i}, \underline{\epsilon}_{i}\right)$ $\in \mathbf{k}$ let us consider

$$
\begin{aligned}
& \|\pi(\underline{u}, \underline{v}, \underline{\epsilon}) \xi\|^{2} \\
& =\sum_{i, j=1}^{N} \overline{c_{i}} c_{j}\left\langle\pi(\underline{u}, \underline{v}, \underline{\epsilon}) \eta\left(\underline{u}_{i}, \underline{v}_{i}, \underline{\epsilon}_{i}\right), \pi(\underline{u}, \underline{v}, \underline{\epsilon}) \eta\left(\underline{u}_{j}, \underline{v}_{j}, \underline{\epsilon}_{j}\right)\right\rangle \\
& =\sum_{i, j=1}^{N} \overline{c_{i}} c_{j}\left\langle\left[\eta\left(\underline{u} \otimes \underline{u}_{i}, \underline{v} \otimes \underline{v}_{i}, \underline{\epsilon} \oplus \underline{\epsilon}_{i}\right)-\left\langle\underline{u}_{i}^{\left(\epsilon_{i}\right)}, \underline{u}_{i}^{\left(\underline{\epsilon}_{i}\right)}\right\rangle \eta(\underline{u}, \underline{v}, \underline{\epsilon})\right],\right. \\
& \left.\left.=\lim _{t \rightarrow 0} \frac{1}{t} \sum_{i, j=1}^{N} \bar{c}_{i} c_{j}\left\langle\underline{u}_{t} \otimes \underline{u}_{j}, \underline{\underline{\epsilon}}\right)\left(\underline{u}^{(\underline{\epsilon})}, \underline{v}_{j}, \underline{\epsilon}\right)\left[\underline{\epsilon}_{j}\right)-\left\langle\underline{u}_{j}^{\left(\underline{\epsilon}_{j}\right)}, \underline{v}_{j}^{\left(\underline{\epsilon}_{j}\right)}\right\rangle \eta(\underline{u}, \underline{v}, \underline{\epsilon})\right]\right\rangle \\
& \left.\quad \times\left(\underline{u}_{i}, \underline{v}_{i}\right) \Omega, U_{t}^{(\underline{\epsilon})}\left(\underline{u}^{(\underline{\epsilon})}, \underline{v}^{(\underline{\epsilon})}\right)\left[U_{t}^{\left(\underline{\epsilon}_{j}\right)}-1\right]\left(\underline{u}_{j}^{\left(\underline{\epsilon}_{j}\right)}, \underline{v}_{j}^{\left(\underline{\epsilon}_{j}\right)}\right) \Omega\right\rangle .
\end{aligned}
$$

In the above identity we have used the fact that for any $\underline{\epsilon} \in \mathbb{Z}_{2}^{m}, \underline{\alpha} \in \mathbb{Z}_{2}^{n}$ and product vectors $\underline{\underline{p}}^{(\underline{\epsilon})}, \underline{w}^{(\underline{\epsilon})} \in \mathbf{h}^{(\underline{\epsilon})}, \underline{x}^{(\underline{\alpha})}, \underline{\mathrm{y}}^{(\underline{\alpha})} \in \mathbf{h}^{(\underline{\alpha})}$

$$
\begin{aligned}
& {\left[U_{t}^{\epsilon} \oplus \underline{\alpha}-1\right]\left(\underline{p}^{(\underline{\epsilon})} \otimes \underline{\mathrm{x}}^{(\underline{\alpha})}, \underline{w}^{(\underline{\epsilon})} \otimes \underline{\mathrm{y}}^{(\underline{\alpha})}\right)-\left\langle\underline{\mathrm{x}}^{(\underline{\alpha})}, \underline{\mathrm{y}}^{(\underline{\alpha})}\right\rangle\left[U_{t}^{(\underline{\epsilon})}-1\right]\left(\underline{p}^{(\underline{\epsilon})}, \underline{w}^{(\underline{\epsilon})}\right)} \\
& =U_{t}^{(\underline{\epsilon})}\left(\underline{p}^{(\underline{\epsilon})}, \underline{w}^{(\underline{\epsilon})}\right)\left[U_{t}^{(\underline{\alpha})}-1\right]\left(\underline{\mathrm{x}}^{(\underline{\alpha})}, \underline{\mathrm{y}}^{(\underline{\alpha})}\right) .
\end{aligned}
$$


Now setting $\phi(t):=\sum_{i,=1}^{N} c_{i}\left[U_{t}^{\left(\epsilon_{i}\right)}-1\right]\left(\underline{u}_{i}^{\left(\epsilon_{i}\right)}, \underline{v}_{i}^{\left(\epsilon_{i}\right)}\right) \Omega \in \mathcal{H}$. we get

$$
\|\pi(\underline{u}, \underline{v}, \underline{\epsilon}) \xi\|^{2}=\lim _{t \rightarrow 0} \frac{1}{t}\left\|U_{t}^{(\underline{\epsilon})}\left(\underline{u}^{(\underline{\epsilon})}, \underline{v}^{(\underline{\epsilon})}\right) \phi(t)\right\|^{2} .
$$

Since $U_{t}^{(\underline{\epsilon})}\left(\underline{u}^{(\underline{\epsilon})}, \underline{v}^{(\epsilon)}\right)$ has its norm bounded by $\|\underline{u}\|^{2}\|\underline{v}\|^{2}$ we get

$$
\begin{aligned}
& \|\pi(\underline{u}, \underline{v}, \underline{\epsilon}) \xi\|^{2} \leq\|\underline{u}\|^{2}\|\underline{v}\|^{2} \lim _{t \rightarrow 0} \frac{1}{t}\|\phi(t)\|^{2} \\
& =\sum_{i, j=1}^{N} \bar{c}_{i} c_{j} \lim _{t \rightarrow 0} \frac{1}{t}\left\langle\left[U_{t}^{\left(\underline{\epsilon}_{i}\right)}-1\right]\left(\underline{u}_{i}^{\left(\underline{\epsilon}_{i}\right)}, \underline{v}_{i}^{\left(\underline{\epsilon}_{i}\right)}\right) \Omega,\left[U_{t}^{\left(\underline{\epsilon}_{j}\right)}-1\right]\left(\underline{u}_{j}^{\left(\underline{\epsilon}_{j}\right)}, \underline{v}_{j}^{\left(\underline{\epsilon}_{j}\right)}\right) \Omega\right\rangle \\
& =\|\underline{u}\|^{2}\|\underline{v}\|^{2}\|\xi\|^{2}
\end{aligned}
$$

which proves that $\pi(\underline{u}, \underline{v}, \underline{\epsilon})$ extends to a bounded operator on $\mathbf{k}$ with $\|\pi(\underline{u}, \underline{v}, \underline{\epsilon})\| \leq\|\underline{u}\|\|\underline{v}\|$.

In order to prove that $\pi$ is a $*$-representation of the algebra $M$ it is enough to show that for any $\underline{\epsilon} \in \mathbb{Z}_{2}^{m}, \underline{\epsilon}^{\prime} \in \mathbb{Z}_{2}^{n}, \underline{\epsilon}^{\prime \prime} \in \mathbb{Z}_{2}^{q}$ and product vectors $\underline{p}, \underline{w} \in$ $\mathbf{h}^{\otimes m}, \underline{p}^{\prime}, \underline{w}^{\prime} \in \mathbf{h}^{\otimes n}, \underline{\mathrm{x}}, \underline{\mathrm{y}} \in \mathbf{h}^{\otimes q}$,

$$
\pi(\underline{u}, \underline{v}, \underline{\epsilon}) \pi\left(\underline{p}, \underline{w}, \underline{\epsilon}^{\prime}\right) \eta\left(\underline{\mathrm{x}}, \underline{\mathrm{y}}, \underline{\epsilon}^{\prime \prime}\right)=\pi\left(\underline{u} \otimes \underline{p}, \underline{v} \otimes \underline{w}, \underline{\epsilon} \oplus \underline{\epsilon}^{\prime}\right) \eta\left(\underline{\mathrm{x}}, \underline{\mathrm{y}}, \underline{\epsilon}^{\prime \prime}\right),
$$

(7.8) $\left\langle\pi(\underline{u}, \underline{v}, \underline{\epsilon}) \eta\left(\underline{p}, \underline{w}, \underline{\epsilon}^{\prime}\right), \eta\left(\underline{\mathrm{x}}, \underline{\mathrm{y}}, \underline{\epsilon}^{\prime \prime}\right)\right\rangle=\left\langle\eta\left(\underline{p}, \underline{w}, \underline{\epsilon}^{\prime}\right), \pi\left(\underline{u}, \underline{v}, \underline{\epsilon}^{*}\right) \eta\left(\underline{\mathrm{x}}, \underline{\mathrm{y}}, \underline{\epsilon}^{\prime \prime}\right)\right\rangle$.

By the definition of $\pi$

$$
\begin{aligned}
& \pi(\underline{u}, \underline{v}, \underline{\epsilon}) \pi\left(\underline{p}, \underline{w}, \underline{\epsilon}^{\prime}\right) \eta\left(\underline{\mathrm{x}}, \underline{\mathrm{y}}, \underline{\epsilon}^{\prime \prime}\right) \\
& =\pi(\underline{u}, \underline{v}, \underline{\epsilon})\left[\eta\left(\underline{p} \otimes \underline{\mathrm{x}}, \underline{w} \otimes \underline{\mathrm{y}}, \underline{\epsilon}^{\prime} \oplus \underline{\epsilon}^{\prime \prime}\right)-\langle\underline{\mathrm{x}}, \underline{\mathrm{y}}\rangle \eta\left(\underline{p}, \underline{w}, \underline{\epsilon}^{\prime}\right)\right] \\
& =\eta\left(\underline{u} \otimes \underline{p} \otimes \underline{\mathrm{x}}, \underline{v} \otimes \underline{w} \otimes \underline{\mathrm{y}}, \underline{\epsilon} \oplus \underline{\epsilon}^{\prime} \oplus \underline{\epsilon}^{\prime \prime}\right)-\langle\underline{p} \otimes \underline{\mathrm{x}}, \underline{w} \otimes \underline{\mathrm{y}}\rangle \eta(\underline{u}, \underline{v}, \underline{\epsilon}) \\
& -\langle\underline{\mathrm{x}}, \underline{\mathrm{y}}\rangle\left[\eta\left(\underline{u} \otimes \underline{p}, \underline{v} \otimes \underline{w}, \underline{\epsilon} \oplus \underline{\epsilon}^{\prime}\right)-\langle\underline{p}, \underline{w}\rangle \eta(\underline{u}, \underline{v}, \underline{\epsilon})\right] \\
& \left.=\eta\left(\underline{u} \otimes \underline{p} \otimes \underline{\mathrm{x}}, \underline{v} \otimes \underline{w} \otimes \underline{\mathrm{y}}, \underline{\epsilon} \oplus \underline{\epsilon}^{\prime} \oplus \underline{\epsilon}^{\prime \prime}\right)-\langle\underline{\mathrm{x}}, \underline{\mathrm{y}}\rangle \eta \underline{u} \otimes \underline{p}, \underline{v} \otimes \underline{w}, \underline{\epsilon} \oplus \underline{\epsilon}^{\prime}\right)
\end{aligned}
$$

and (7.7) follows. To see (7.8) let us look at the left hand side. By (7.6)

$$
\begin{aligned}
& \left\langle\pi(\underline{u}, \underline{v}, \underline{\epsilon}) \eta\left(\underline{p}, \underline{w}, \underline{\epsilon}^{\prime}\right), \eta\left(\underline{\mathrm{x}}, \underline{\mathrm{y}}, \underline{\epsilon}^{\prime \prime}\right)\right\rangle \\
& =\lim _{t \rightarrow 0} \frac{1}{t}\left\langle U_{t}^{(\underline{\epsilon})}(\underline{u}, \underline{v})\left(U_{t}^{\epsilon^{\prime}}-1\right)(\underline{p}, \underline{w}) \Omega,\left(U_{\underline{\epsilon}^{\prime \prime}}-1\right)(\underline{\mathrm{x}}, \underline{\mathrm{y}}) \Omega\right\rangle \\
& =\lim _{t \rightarrow 0} \frac{1}{t}\left\langle\left(U_{t}^{\underline{\epsilon}^{\prime}}-1\right)(\underline{p}, \underline{w}) \Omega, U_{t}^{\underline{\epsilon}^{*}}(\underline{v}, \underline{u})\left(U_{t}^{\epsilon^{\prime \prime}}-1\right)(\underline{\mathrm{x}}, \underline{\mathrm{y}}) \Omega\right\rangle \\
& =\left\langle\eta\left(\underline{p}, \underline{w}, \underline{\epsilon}^{\prime}\right), \pi\left(\underline{v}, \underline{u}, \underline{\epsilon}^{*}\right) \eta\left(\underline{\mathrm{x}}, \underline{\mathrm{y}}, \underline{\epsilon}^{\prime \prime}\right)\right\rangle=R H S .
\end{aligned}
$$


Thus

$$
\begin{aligned}
& \pi(\underline{u}, \underline{v}, \underline{\epsilon}) \pi\left(\underline{p}, \underline{w}, \underline{\epsilon}^{\prime}\right)=\pi\left(\underline{u} \otimes \underline{p}, \underline{v} \otimes \underline{w}, \underline{\epsilon} \oplus \underline{\epsilon}^{\prime}\right) \\
& \pi(\underline{u}, \underline{v}, \underline{\epsilon})^{*}=\pi\left(\underline{v}, \underline{u}, \underline{\epsilon}^{*}\right) .
\end{aligned}
$$

Lemma 7.2. (i) For any $u, v \in \mathbf{h}, \eta(u, v, 1)=-\eta(u, v, 0)$.

(ii) For any $(\underline{u}, \underline{v}, \underline{\epsilon}) \in M_{0}, \underline{u}=\otimes_{i=1}^{n} u_{i}, \underline{v}=\otimes_{i=1}^{n} v_{i}$ and $\underline{\epsilon}=\left(\epsilon_{1}, \epsilon_{2}, \cdots, \epsilon_{n}\right)$

$$
\eta(\underline{u}, \underline{v}, \underline{\epsilon})=\sum_{i=1}^{n}(-1)^{\epsilon_{i}} \prod_{k \neq i}\left\langle u_{k}, v_{k}\right\rangle \eta\left(u_{i}, v_{i}, \epsilon_{i}\right)
$$

Proof. (i) Follows from the identity (6.8).

(ii) For any $\left(\underline{p}, \underline{w}, \underline{\epsilon}^{\prime}\right) \in M_{0}$, by $(7.4)$ and Lemma 6.6 , we have

$$
\begin{aligned}
& \left\langle\eta(\underline{u}, \underline{v}, \underline{\epsilon}), \eta\left(\underline{p}, \underline{w}, \underline{\epsilon}^{\prime}\right)\right\rangle=K\left((\underline{u}, \underline{v}, \underline{\epsilon}),\left(\underline{p}, \underline{w}, \underline{\epsilon}^{\prime}\right)\right) \\
& =\lim _{t \rightarrow 0} \frac{1}{t}\left\langle\left(U_{t}^{(\underline{\epsilon})}-1\right)(\underline{u}, \underline{v}) \Omega,\left(U_{\underline{\epsilon}^{\prime}}-1\right)(\underline{p}, \underline{w}) \Omega\right\rangle \\
& =\sum_{i=1}^{n} \prod_{k \neq i} \overline{\left\langle u_{k}, v_{k}\right\rangle} \lim _{t \rightarrow 0} \frac{1}{t}\left\langle\left(U_{t}-1\right)^{\left(\epsilon_{i}\right)}\left(u_{i}, v_{i}\right) \Omega,\left(U_{t}^{\epsilon^{\prime}}-1\right)(\underline{p}, \underline{w}) \Omega\right\rangle \\
& =\sum_{i=1}^{n} \prod_{k \neq i} \overline{\left\langle u_{k}, v_{k}\right\rangle}\left\langle\eta\left(u_{i}, v_{i}, \epsilon_{i}\right), \eta\left(\underline{p}, \underline{w}, \underline{\epsilon^{\prime}}\right)\right\rangle .
\end{aligned}
$$

Since $\left\{\eta\left(\underline{p}, \underline{w}, \underline{\epsilon}^{\prime}\right):\left(\underline{p}, \underline{w}, \underline{\epsilon}^{\prime}\right) \in M_{0}\right\}$ is a total subset of $\mathbf{k}$, by the part (i) requirement follows.

Remark 7.3. Writing $\eta(u, v)$ for the vector $\eta(u, v, 0) \in \mathbf{k}$,

$$
\overline{\operatorname{Span}}\{\eta(u, v): u, v \in \mathbf{h}\}=\mathbf{k} \text {. }
$$

Remark 7.4. The $*$-representation $\pi$ of $M$ in $\mathbf{k}$ is trivial

$$
\pi(\underline{u}, \underline{v}, \underline{\epsilon}) \eta\left(\underline{p}, \underline{w}, \underline{\epsilon}^{\prime}\right)=\langle\underline{u}, \underline{v}\rangle \eta\left(\underline{p}, \underline{w}, \underline{\epsilon}^{\prime}\right)
$$

Now we fix an ONB $\left\{E_{j}: j \geq 1\right\}$ for the separable Hilbert space $\mathbf{k}$. Then we have the following crucial observations.

Lemma 7.5. (i) There exists a unique family $\left\{L_{j}: j \geq 1\right\}$ in $\mathcal{B}(\mathbf{h})$ such that $\left\langle u, L_{j} v\right\rangle=\left\langle E_{j}, \eta(u, v)\right\rangle$ and $\sum_{j \geq 1}\left\|L_{j} u\right\|^{2} \leq 2\|G\|\|u\|^{2}, \forall u \in \mathbf{h}$, so that $\sum_{j \geq 1} L_{j}^{*} L_{j}$ converges strongly. 
(ii) The family of operators $\left\{L_{j}: j \geq 1\right\}$ is linearly independent, i.e. $\sum_{j \geq 1} c_{j} L_{j}$ $=0$ for some $c=\left(c_{j}\right) \in l^{2}(\mathbb{N})$ implies $c_{j}=0, \forall j$.

(iii) If we set $i H:=G+\frac{1}{2} \sum_{j \geq 1} L_{j}^{*} L_{j}$ then $H$ is a bounded self-adjoint operator on $\mathbf{h}$.

Proof. (i) By (7.5), for any $u, v \in \mathbf{h}$

$$
\begin{aligned}
& \|\eta(u, v)\|^{2} \\
& =\langle u, \mathcal{L}(|v\rangle\langle v|) u\rangle-\overline{\langle u, v\rangle}\langle u, G v\rangle-\overline{\langle u, G v\rangle}\langle u, v\rangle \\
& \leq[\|\mathcal{L}\|+2\|G\|]\|u\|^{2}\|v\|^{2} .
\end{aligned}
$$

So for each $j \geq 1$, the map $\eta_{j}(u, v):=\left\langle E_{j}, \eta(u, v)\right\rangle$, defines a bounded quadratic form on $\mathbf{h}$ and hence by Riesz's representation theorem there exists a unique bounded operator $L_{j} \in \mathcal{B}(\mathbf{h})$ such that $\left\langle u, L_{j} v\right\rangle=\eta_{j}(u, v)$. Now consider the following

$$
\begin{aligned}
& \sum_{j}\left\|L_{j} u\right\|^{2}=\sum_{j, k}\left|\eta_{j}\left(e_{k}, u\right)\right|^{2}=\sum_{k}\left\|\eta\left(e_{k}, u\right)\right\|^{2} \\
& =\sum_{k}\left[\left\langle e_{k}, \mathcal{L}(|u\rangle\langle u|) e_{k}\right\rangle-\overline{\left\langle e_{k}, u\right\rangle}\left\langle e_{k}, G u\right\rangle-\overline{\left\langle e_{k}, G u\right\rangle}\left\langle e_{k}, u\right\rangle\right] \\
& =\operatorname{Tr} \mathcal{L}(|u\rangle\langle u|)-\langle u, G u\rangle-\overline{\langle u, G u\rangle .}
\end{aligned}
$$

Since $Z_{t}$ is trace preserving

$$
\sum_{j}\left\|L_{j} u\right\|^{2}=-\langle u, G u\rangle-\overline{\langle u, G u\rangle} \leq 2\|G\|\|u\|^{2} .
$$

(ii) Let $\sum_{j \geq 1} c_{j} L_{j}=0$ for some $c=\left(c_{j}\right) \in l^{2}(\mathbb{N})$. Then for any $u, v \in \mathbf{h}$ we have

$$
0=\left\langle u, \sum_{j \geq 1} c_{j} L_{j} v\right\rangle=\sum_{j \geq 1} c_{j}\left\langle u, L_{j} v\right\rangle=\left\langle\sum_{j \geq 1} \bar{c}_{j} E_{j}, \eta(u, v)\right\rangle .
$$

Since $\overline{\operatorname{Span}}\{\eta(u, v): u, v \in \mathbf{h}\}=\mathbf{k}$, it follows that $\sum_{j \geq 1} \bar{c}_{j} E_{j}=0 \in \mathbf{k}$ and hence $c_{j}=0, \forall j$.

(iii) The boundedness of $G$ and (7.13) imply that $\sum_{j \geq 1} L_{j}^{*} L_{j}$ is a bounded self-adjoint operator and hence $H$ is bounded. For any $u \in \mathbf{h}$ by the identity (7.13)

$$
\begin{aligned}
& \left\langle u,\left(2 G+\sum_{j \geq 1} L_{j}^{*} L_{j}\right) u\right\rangle \\
& =\langle u, 2 G u\rangle+\sum_{j}\left\|L_{j} u\right\|^{2}=\langle u, G u\rangle-\langle G u, u\rangle \\
& =-\left\langle\left(2 G+\sum_{j \geq 1} L_{j}^{*} L_{j}\right) u, u\right\rangle
\end{aligned}
$$


Thus $\langle u, H u\rangle=\langle H u, u\rangle$ and by applying the polarization principle to the sesqui-linear form $(u, v) \mapsto\langle u, H u\rangle$ it proves that $H$ is self-adjoint.

Lemma 7.6. The generator $\mathcal{L}$ of the uniformly continuous semigroup $Z_{t}$ on $\mathcal{B}_{1}(\mathbf{h})$ satisfies

$$
\mathcal{L} \rho=G \rho+\rho G^{*}+\sum_{j \geq 1} L_{j} \rho L_{j}^{*}, \quad \forall \rho \in \mathcal{B}_{1}(\mathbf{h})
$$

Proof. By (7.5), for any $u, v, p, w \in \mathbf{h}$ we have

$$
\begin{aligned}
& \langle\eta(u, v), \eta(p, w)\rangle=\sum_{j \geq 1} \overline{\left\langle u, L_{j} v\right\rangle}\left\langle p, L_{j} w\right\rangle \\
& =\langle p, \mathcal{L}(|w\rangle\langle v|) u\rangle-\overline{\langle u, v\rangle}\langle p, G w\rangle-\overline{\langle u, G v\rangle}\langle p, w\rangle,
\end{aligned}
$$

which gives

$$
\begin{aligned}
& \langle p, \mathcal{L}(|w><v|) u\rangle \\
& =\langle p,|G w><v| u\rangle+\langle p,|w><G v| u\rangle+\sum_{j \geq 1}\left\langle p,\left|L_{j} w><L_{j} v\right| u\right\rangle \\
& =\langle p, G|w><v| u\rangle+\left\langle p,|w><v| G^{*} u\right\rangle+\sum_{j \geq 1}\left\langle p, L_{j}|w><v| L_{j}^{*} u\right\rangle .
\end{aligned}
$$

Since all the operators involved are bounded (7.14) follows.

\section{§8. Associated Hudson-Parthasarathy (HP) Flows}

Recall from the previous section that starting from the family of unitary operators $\left\{U_{s, t}\right\}$ with Assumption $\mathbf{A}, \mathbf{B}$ and $\mathbf{C}$ we obtained the noise Hilbert space $\mathbf{k}$ and bounded linear operators $G, L_{j}: j \geq 1$ on the initial Hilbert space h. Now define a family of operators $\left\{L_{\nu}^{\mu}: \mu, \nu \geq 0\right\}$ in $\mathcal{B}(\mathbf{h})$ by

$$
L_{\nu}^{\mu}= \begin{cases}G=i H-\frac{1}{2} \sum_{k \geq 1} L_{k}^{*} L_{k} & \text { for }(\mu, \nu)=(0,0) \\ L_{j} & \text { for }(\mu, \nu)=(j, 0) \\ -L_{k}^{*} & \text { for }(\mu, \nu)=(0, k) \\ 0 & \text { for }(\mu, \nu)=(j, k) .\end{cases}
$$

Note that the indices $\mu, \nu$ vary over non negative integers while $j, k$ vary over non zero positive integers. 
Let us consider the HP type quantum stochastic differential equation in $\mathbf{h} \otimes \Gamma\left(L^{2}\left(\mathbb{R}_{+}, \mathbf{k}\right)\right):$

$$
V_{s, t}=1_{\mathbf{h} \otimes \Gamma}+\sum_{\mu, \nu \geq 0} \int_{s}^{t} V_{s, r} L_{\nu}^{\mu} \Lambda_{\mu}^{\nu}(d r)
$$

with bounded operator coefficients $L_{\nu}^{\mu}$ given by (8.1). By Theorem 2.2, there exists a unique unitary solution $\left\{V_{s, t}\right\}$ of the above HP equation. We shall write $V_{t}:=V_{0, t}$ for simplicity. The family $\left\{V_{s, t}^{*}\right\}$ satisfies:

$$
d V_{s, t}^{*}=\sum_{\mu, \nu \geq 0}\left(L_{\mu}^{\nu}\right)^{*} V_{s, t}^{*} \Lambda_{\mu}^{\nu}(d t), V_{s, s}=1_{\mathbf{h} \otimes \Gamma}
$$

and for any $u, v \in \mathbf{h}, V_{s, t}(u, v)$ and $V_{s, t}(u, v)^{*}$ satisfy the following qsde on $\Gamma$ :

$$
\begin{gathered}
d V_{s, t}(u, v)=\sum_{\mu, \nu \geq 0} V_{s, t}\left(u, L_{\nu}^{\mu} v\right) \Lambda_{\mu}^{\nu}(d t), V_{s, s}(u, v)=\langle u, v\rangle 1_{\Gamma} . \\
\left.d V_{s, t}^{*}(u, v)=\sum_{\mu, \nu \geq 0} V_{s, t}^{*}\left(L_{\mu}^{\nu} u, v\right) \Lambda_{\mu}^{\nu}(d t), V_{s, s}^{*}(u, v)\right]=\langle u, v\rangle 1_{\Gamma} .
\end{gathered}
$$

As for the family of unitary operators $\left\{U_{s, t}\right\}$ on $\mathbf{h} \otimes \mathcal{H}$, for $\underline{\epsilon}=\left(\epsilon_{1}, \epsilon_{2}, \cdots, \epsilon_{n}\right) \in$ $\mathbb{Z}_{2}^{n}$ we define $V_{s, t}^{(\underline{\epsilon})} \in \mathcal{B}\left(\mathbf{h}^{\otimes n} \otimes \Gamma\right)$ by setting $V_{s, t}^{(\epsilon)} \in \mathcal{B}(\mathbf{h} \otimes \bar{\Gamma})$ by

$$
\begin{gathered}
V_{s, t}^{(\epsilon)}=V_{s, t} \text { for } \epsilon=0 \\
=V_{s, t}^{*} \text { for } \epsilon=1 .
\end{gathered}
$$

We shall write $V_{s, t}^{(n)}$ for $V_{s, t}^{(\underline{\epsilon})}, \underline{\epsilon}=(0,0, \cdots, 0) \in \mathbb{Z}_{2}^{n}$.

Lemma 8.1. The family of unitary operators $\left\{V_{s, t}\right\}$ satisfies

(i) For any $0 \leq r \leq s \leq t<\infty, V_{r, t}=V_{r, s} V_{s, t}$.

(ii) For $[q, r) \cap[s, t)=\varnothing, V_{q, r}(u, v)$ commute with $V_{s, t}(p, w)$ and $V_{s, t}(p, w)^{*}$ for every $u, v, p, w \in \mathbf{h}$.

(iii) For any $0 \leq s \leq t<\infty$,

$\left\langle\boldsymbol{e}(0), V_{s, t}(u, v) \boldsymbol{e}(0)\right\rangle=\left\langle\boldsymbol{e}(0), V_{t-s}(u, v) \boldsymbol{e}(0)\right\rangle=\left\langle u, T_{t-s} v\right\rangle, \forall u, v \in \mathbf{h}$.

Proof. (i) For fixed $0 \leq r \leq s \leq t<\infty$, we set $W_{r, t}=V_{r, s} V_{s, t}$ and $W_{r, s}=V_{r, s}$. Then by (8.2) we have 


$$
\begin{aligned}
& W_{r, t}=V_{r, s}+\sum_{\mu, \nu \geq 0} \int_{s}^{t} V_{r, s} V_{s, q} L_{\nu}^{\mu} \Lambda_{\mu}^{\nu}(d q) \\
& =W_{r, s}+\sum_{\mu, \nu \geq 0} \int_{s}^{t} W_{r, q} L_{\nu}^{\mu} \Lambda_{\mu}^{\nu}(d q) .
\end{aligned}
$$

Thus the family of unitary operators $\left\{W_{r, t}\right\}$ also satisfies the HP equation (8.2) and, hence by uniqueness of the solution of this qsde, $W_{r, t}=V_{r, t}, \forall t \geq s$ and the result follows.

(ii) For any $0 \leq s \leq t<\infty V_{s, t} \in \mathcal{B}\left(\mathbf{h} \otimes \Gamma_{[s, t]}\right)$. So for $p, w \in \mathbf{h}, V_{s, t}(p, w) \in$ $\mathcal{B}\left(\Gamma_{[s, t]}\right)$ and the statement follows.

(iii) Let us set a family of contraction operators $\left\{\widetilde{S}_{s, t}\right\}$ on $\mathbf{h}$ by

$$
\left\langle u, \widetilde{S}_{s, t} v\right\rangle=\left\langle u \otimes \mathbf{e}(0), V_{s, t} v \otimes \mathbf{e}(0)\right\rangle, \forall u, v \in \mathbf{h} .
$$

Then for fixed $s \geq 0$, this family $\left\{\widetilde{S}_{s, t}\right\}$ satisfies the following differential equation

$$
\frac{d \widetilde{S}_{s, t}}{d t}=\widetilde{S}_{s, t} G
$$

where $G\left(=L_{0}^{0}\right)$ is the generator of the uniformly continuous semigroup $\left\{T_{t}\right\}$ so $\widetilde{S}_{s, t}=T_{t-s}$ and this proves the claim.

Consider the family of maps $\widetilde{Z}_{s, t}$ defined by

$$
\widetilde{Z}_{s, t} \rho=\operatorname{Tr}_{\mathcal{H}}\left[V_{s, t}(\rho \otimes|\mathbf{e}(0)><\mathbf{e}(0)|) V_{s, t}^{*}\right], \forall \rho \in \mathcal{B}_{1}(\mathbf{h}) .
$$

As for $Z_{t}$, it can be easily seen that $\widetilde{Z}_{s, t}$ is a contractive family of maps on $\mathcal{B}_{1}(\mathbf{h})$ and in particular, for any $u, v, p, w \in \mathbf{h}$

$$
\left\langle p, \widetilde{Z}_{s, t}(|w><v|) u\right\rangle=\left\langle V_{s, t}(u, v) \mathbf{e}(0), V_{s, t}(p, w) \mathbf{e}(0)\right\rangle .
$$

Lemma 8.2. The family $\widetilde{Z}_{t}:=\widetilde{Z}_{0, t}$ is a uniformly continuous semigroup of contractions on $\mathcal{B}_{1}(\mathbf{h})$ and $\widetilde{Z}_{s, t}=\widetilde{Z}_{t-s}=Z_{t-s}$. 
Proof. By (8.4) and Ito's formula

$\left\langle p,\left[\widetilde{Z}_{s, t}-1\right](|w><v|) u\right\rangle$

$=\left\langle V_{s, t}(u, v) \mathbf{e}(0), V_{s, t}(p, w) \mathbf{e}(0)\right\rangle-\overline{\langle u, v\rangle}\langle p, w\rangle$

$=\int_{s}^{t}\left\langle V_{s, \tau}(u, v) \mathbf{e}(0), V_{s, \tau}(p, G w) \mathbf{e}(0)\right\rangle d \tau+\int_{s}^{t}\left\langle V_{s, \tau}(u, G v) \mathbf{e}(0), V_{s, \tau}(p, w) \mathbf{e}(0)\right\rangle d \tau$

$+\int_{s}^{t}\left\langle V_{s, \tau}\left(u, L_{j} v\right) \mathbf{e}(0), V_{s, \tau}\left(p, L_{j} w\right) \mathbf{e}(0)\right\rangle d \tau$

$=\int_{s}^{t}\left\langle p, \widetilde{Z}_{s, \tau}(|G w><v|) u\right\rangle d \tau+\int_{s}^{t}\left\langle p, \widetilde{Z}_{s, \tau}(|w><G v|) u\right\rangle d \tau$

$+\sum_{j \geq 1} \int_{s}^{t}\left\langle p, \widetilde{Z}_{s, \tau}\left(\left|L_{j} w><L_{j} v\right|\right) u\right\rangle d \tau$

$=\int_{s}^{t}\left\langle p, \widetilde{Z}_{s, \tau} \mathcal{L}(|w><v|) u\right\rangle d \tau$,

where $\mathcal{L}$ is the generator of the uniformly continuous semigroup $Z_{t}$. Since the maps $\mathcal{L}$ and $\widetilde{Z}_{a, b}: 0 \leq a \leq b$ are bounded, for fixed $s \geq 0, \widetilde{Z}_{s, t}$ satisfies the differential equation

$$
\widetilde{Z}_{s, t}(\rho)=\rho+\int_{s}^{t} \widetilde{Z}_{s, \tau} \mathcal{L}(\rho) d \tau, \quad \rho \in \mathcal{B}_{1}(\mathbf{h}) .
$$

Hence $\widetilde{Z}_{t}$ is a uniformly continuous semigroup on $\mathcal{B}_{1}(\mathbf{h})$ and $\widetilde{Z}_{s, t}=\widetilde{Z}_{t-s}=$ $Z_{t-s}$.

\section{$\S 9 . \quad$ Minimality of HP Flows}

In this section we shall show the minimality of the HP flow $V_{s, t}$ discussed above. We prove that the subset $\mathcal{S}^{\prime}:=\left\{\zeta=V_{\underline{s}, t}(\underline{u}, \underline{v}) \mathbf{e}(0):=V_{s_{1}, t_{1}}\left(u_{1}, v_{1}\right) \cdots\right.$ $V_{s_{n}, t_{n}}\left(u_{n}, v_{n}\right) \mathbf{e}(0): \underline{s}=\left(s_{1}, s_{2}, \cdots, s_{n}\right), \underline{t}=\left(t_{1}, t_{2}, \cdots, t_{n}\right): 0 \leq s_{1} \leq t_{1} \leq$ $\left.s_{2} \leq \ldots \leq s_{n} \leq t_{n}<\infty, n \geq 1, \underline{u}=\otimes_{i=1}^{n} u_{i}, \underline{v}=\otimes_{i=1}^{n} v_{i} \in \mathbf{h}^{\otimes n}\right\}$ is total in the symmetric Fock space $\Gamma\left(L^{2}\left(\mathbb{R}_{+}, \mathbf{k}\right)\right)$.

We note that for any $0 \leq s<t \leq \tau<\infty, u, v \in \mathbf{h}$ by the HP equation

$$
\begin{aligned}
& \frac{1}{t-s}\left[V_{s, t}-1\right](u, v) \mathbf{e}(0) \\
& =\frac{1}{t-s}\left\{\sum_{j \geq 1} \int_{s}^{t} V_{s, \lambda}\left(u, L_{j} v\right) a_{j}^{\dagger}(d \lambda)+\int_{s}^{t} V_{s, \lambda}(u, G v) d \lambda\right\} \mathbf{e}(0) \\
& =\gamma(s, t, u, v)+\langle u, G v\rangle \mathbf{e}(0)+\zeta(s, t, u, v)+\varsigma(s, t, u, v)
\end{aligned}
$$

where 


$$
\begin{aligned}
\gamma(s, t, u, v) & :=\frac{1}{t-s} \sum_{j \geq 1}\left\langle u, L_{j} v\right\rangle a_{j}^{\dagger}([s, t]) \mathbf{e}(0) \\
\zeta(s, t, u, v) & :=\frac{1}{t-s} \sum_{j \geq 1} \int_{s}^{t}\left(V_{s, \lambda}-1\right)\left(u, L_{j} v\right) a_{j}^{\dagger}(d \lambda) \mathbf{e}(0) \\
\varsigma(s, t, u, v) & :=\frac{1}{t-s} \int_{s}^{t}\left(V_{s, \lambda}-1\right)(u, G v) d \lambda \mathbf{e}(0) .
\end{aligned}
$$

Note that any $\xi \in \Gamma$ can be written as $\xi=\xi^{(0)} \mathbf{e}(0) \oplus \xi^{(1)} \oplus \cdots$, where $\xi^{(n)}$ is in the $n$-fold symmetric tensor product $L^{2}\left(\mathbb{R}_{+}, \mathbf{k}\right)^{\otimes n} \equiv L^{2}\left(\Sigma_{n}\right) \otimes \mathbf{k}^{\otimes n}$, where $\Sigma_{n}$ is the $n$-simplex $\left\{\underline{t}=\left(t_{1}, t_{2}, \cdots, t_{n}\right): 0 \leq t_{1}<t_{2}<\ldots<t_{n}<\infty\right\}$.

Lemma 9.1. Let $\tau \geq 0$. For any $v \in \mathbf{h}, 0 \leq s \leq t \leq \tau$, define constants $C_{\tau}=2 e^{\tau}$ and $C_{\tau, v}=C_{\tau}\left\{\sum_{j \geq 1}\left\|L_{j} v\right\|^{2}+\tau\|G v\|^{2}\right\}$. Then

(i)

$$
\left\|\left(V_{s, t}-1\right) v e(0)\right\|^{2} \leq C_{\tau, v}(t-s) .
$$

(ii) For any $u \in \mathbf{h}$

$$
\begin{aligned}
& \left\|\sum_{j \geq 1} \int_{s}^{t} V_{s, \lambda}\left(u, L_{j} v\right) a_{j}^{\dagger}(d \lambda) \boldsymbol{e}(0)\right\|^{2} \\
& \leq C_{\tau}\|u\|^{2} \sum_{j \geq 1} \int_{s}^{t}\left\|V_{s, \lambda} L_{j} v \otimes \boldsymbol{e}(0)\right\|^{2} d \lambda \\
& \leq C_{\tau}(t-s)\|u\|^{2} \sum_{j \geq 1}\left\|L_{j} v\right\|^{2} .
\end{aligned}
$$

Proof. (i) By estimates of quantum stochastic integration (Proposition $27.1,[16])$

$$
\begin{aligned}
& \left\|\left(V_{s, t}-1\right) v \mathbf{e}(0)\right\|^{2} \\
& =\left\|\sum_{j \geq 1} \int_{s}^{t} V_{s, \lambda} L_{j} a_{j}^{\dagger}(d \lambda) \quad v \mathbf{e}(0)+\int_{s}^{t} V_{s, \lambda} G d \lambda v \mathbf{e}(0)\right\|^{2} \\
& \leq C_{\tau} \int_{s}^{t}\left\{\sum_{j \geq 1}\left\|L_{j} v\right\|^{2}+\|G v\|^{2}\right\} d \lambda \\
& =C_{\tau, v}(t-s) .
\end{aligned}
$$


(ii) For any $\phi$ in the Fock space $\Gamma\left(L^{2}\left(\mathbb{R}_{+}, \mathbf{k}\right)\right)$,

$$
\begin{aligned}
& \left.\left\langle\phi, \sum_{j \geq 1} \int_{s}^{t} V_{s, \lambda}\left(u, L_{j} v\right) a_{j}^{\dagger}(d \lambda) \mathbf{e}(0)\right\rangle\right|^{2} \\
& =\left|\left\langle u \otimes \phi,\left\{\sum_{j \geq 1} \int_{s}^{t} V_{s, \lambda} L_{j} a_{j}^{\dagger}(d \lambda)\right\} v \mathbf{e}(0)\right\rangle\right|^{2} \\
& \leq\|u \otimes \phi\|^{2}\left\|\left\{\sum_{j \geq 1} \int_{s}^{t} V_{s, \lambda} L_{j} a_{j}^{\dagger}(d \lambda)\right\} v \mathbf{e}(0)\right\|^{2} .
\end{aligned}
$$

By estimates of quantum stochastic integration the above quantity is

$$
\leq C_{\tau}\|u \otimes \phi\|^{2} \sum_{j \geq 1} \int_{s}^{t}\left\|V_{s, \lambda} L_{j} v \mathbf{e}(0)\right\|^{2} d \lambda .
$$

Since $\phi$ is arbitrary and the $V_{s, \lambda}$ 's are contractive the statement follows.

Lemma 9.2. Let $\tau \geq 0$. For any $u, v \in \mathbf{h}, 0 \leq s \leq t \leq \tau$

(i) $\left\|\left(V_{s, t}-1\right)(u, v) \quad e(0)\right\|^{2} \leq 2 C_{\tau, v}\|u\|^{2}(t-s)$.

(ii) $\sup \left\{\|\zeta(s, t, u, v)\|^{2}: 0 \leq s \leq t \leq \tau\right\}<\infty$ and

$\|\varsigma(s, t, u, v)\| \leq\|u\| \sqrt{2 C_{\tau, G v}(t-s)}, \forall 0 \leq s<t \leq \tau$.

(iii) For any $\xi \in \Gamma\left(L^{2}\left(\mathbb{R}_{+}, \mathbf{k}\right)\right), \quad \lim _{s \rightarrow t}\langle\xi, \zeta(s, t, u, v)\rangle=0$ and

$$
\lim _{s \rightarrow t}\langle\xi, \gamma(s, t, u, v)\rangle=\sum_{j \geq 1}\left\langle u, L_{j} v\right\rangle \overline{\xi_{j}^{(1)}}(t)=\left\langle\xi^{(1)}(t), \eta(u, v)\right\rangle, \text { a.e. } t \geq 0 .
$$

Proof. (i) By identity (9.1) and Lemma 9.1 (ii) we have

$$
\begin{aligned}
& \left\|\left(V_{s, t}-1\right)(u, v) \mathbf{e}(0)\right\|^{2} \\
& =\left\|\sum_{j \geq 1} \int_{s}^{t} V_{s, \alpha}\left(u, L_{j} v\right) a_{j}^{\dagger}(d \alpha) \mathbf{e}(0)+\int_{s}^{t} V_{s, \alpha}(u, G v) \mathbf{e}(0) d \alpha\right\|^{2} \\
& \leq 2\left\|\sum_{j \geq 1} \int_{s}^{t} V_{s, \alpha}\left(u, L_{j} v\right) a_{j}^{\dagger}(d \alpha) \mathbf{e}(0)\right\|^{2}+\left[\int_{s}^{t}\left\|V_{s, \alpha}(u, G v) \mathbf{e}(0)\right\| d \alpha\right]^{2} \\
& \leq 2\|u\|^{2}\left[C_{\tau}(t-s) \sum_{j \geq 1}\left\|L_{j} v\right\|^{2}+[(t-s)\|G v\|]^{2}\right] \\
& \leq 2 C_{\tau, v}\|u\|^{2}(t-s) .
\end{aligned}
$$


(ii) To prove the first statement, as in the Lemma 9.1 (ii) we consider

$$
\begin{aligned}
& \|\zeta(s, t, u, v)\|^{2}=\frac{1}{(t-s)^{2}}\left\|\sum_{j \geq 1} \int_{s}^{t}\left(V_{s, \lambda}-1\right)\left(u, L_{j} v\right) a_{j}^{\dagger}(d \lambda) \quad \mathbf{e}(0)\right\|^{2} \\
& \leq \frac{\|u\|^{2}}{(t-s)^{2}}\left\|\sum_{j \geq 1} \int_{s}^{t}\right\|\left(V_{s, \lambda}-1\right) L_{j} v \mathbf{e}(0) \|^{2} d \lambda
\end{aligned}
$$

Now by Lemma 9.1 (i), the above quantity is

$$
\begin{aligned}
& \left.\leq \frac{C_{\tau}\|u\|^{2}}{(t-s)^{2}} \sum_{j \geq 1} C_{\tau}(t-s)^{2}\left\{\sum_{i \geq 1}\left\|L_{i} L_{j} v\right\|^{2}+\tau\left\|G L_{j} v\right\|\right]^{2}\right\} \\
& \leq C_{\tau}^{2}\|u\|^{2}\left\{\sum_{j \geq 1} \sum_{i \geq 1}\left\|L_{i} L_{j} v\right\|^{2}+\tau \sum_{j \geq 1}\left\|G L_{j} v\right\|^{2}\right\} .
\end{aligned}
$$

Since $\sum_{j \geq 1}\left\|L_{j} v\right\|^{2}=-2 R e\langle v, G v\rangle$, the above quantity is bounded and is independent of $s, t$.

To prove the second statement consider the following,

$$
\begin{aligned}
& \|\varsigma(s, t, u, v)\|=\frac{1}{(t-s)}\left\|\int_{s}^{t}\left(V_{s, \lambda}-1\right)(u, G v) d \lambda \mathbf{e}(0)\right\| \\
& \leq \frac{1}{(t-s)} \int_{s}^{t}\left\|\left(V_{s, \lambda}-1\right)(u, G v) \mathbf{e}(0)\right\| d \lambda .
\end{aligned}
$$

By (i) the estimate follows.

(iii) To prove the first statement, let us consider the following. For any $f \in$ $L^{2}\left(\mathbb{R}_{+}, \mathbf{k}\right)$,

$$
\begin{aligned}
& \langle\mathbf{e}(f), \zeta(s, t, u, v)\rangle=\left\langle\mathbf{e}(f), \frac{1}{t-s} \sum_{j \geq 1} \int_{s}^{t}\left(V_{s, \lambda}-1\right)\left(u, L_{j} v\right) a_{j}^{\dagger}(d \lambda) \mathbf{e}(0)\right\rangle \\
& =\frac{1}{t-s} \sum_{j \geq 1} \int_{s}^{t} \overline{f_{j}(\lambda)}\left\langle\mathbf{e}(f),\left(V_{s, \lambda}-1\right)\left(u, L_{j} v\right) \mathbf{e}(0)\right\rangle d \lambda \\
& =\frac{1}{t-s} \int_{s}^{t} G(s, \lambda) d \lambda,
\end{aligned}
$$

where $G(s, \lambda)=\sum_{j \geq 1} \overline{f_{j}(\lambda)}\left\langle\mathbf{e}(f),\left(V_{s, \lambda}-1\right)\left(u, L_{j} v\right) \quad \mathbf{e}(0)\right\rangle$. Note that the complex valued function $G(s, \lambda)$ is uniformly continuous in both the variables $s, \lambda$ on $[0, \tau]$ and $G(t, t)=0$. So we get

$$
\lim _{s \rightarrow t}\langle\mathbf{e}(f), \zeta(s, t, u, v)\rangle=0
$$


Since $\zeta(s, t, u, v)$ is uniformly bounded in $s, t$

$$
\lim _{s \rightarrow t}\langle\xi, \zeta(s, t, u, v)\rangle=0, \forall \xi \in \Gamma
$$

To prove the second statement, we consider

$$
\langle\xi, \gamma(s, t, u, v)\rangle=\frac{1}{t-s} \sum_{j \geq 1}\left\langle u, L_{j} v\right\rangle \int_{s}^{t} \overline{\xi_{j}^{(1)}}(\lambda) d \lambda
$$

Since

$$
\left|\sum_{j \geq 1}\left\langle u, L_{j} v\right\rangle \overline{\xi_{j}^{(1)}}(t)\right|^{2} \leq\|u\|^{2} \sum_{j \geq 1}\left\|L_{j} v\right\|^{2} \sum_{j \geq 1}\left|\xi_{j}^{(1)}(t)\right|^{2} \leq C\|v\|^{2}\left\|\xi^{(1)}(t)\right\|^{2},
$$

the function $\sum_{j \geq 1}\left\langle u, L_{j} v\right\rangle \overline{\xi_{j}^{(1)}}(\cdot)$ is in $L^{2}$ and hence locally integrable. Thus we get

$$
\lim _{s \rightarrow t}\langle\xi, \gamma(s, t, u, v)\rangle=\sum_{j \geq 1}\left\langle u, L_{j} v\right\rangle \overline{\xi_{j}^{(1)}}(t) \quad \text { a.e. } t \geq 0 .
$$

Lemma 9.3. For $n \geq 1, \underline{t} \in \Sigma_{n}$ and $u_{k}, v_{k} \in \mathbf{h}: k=1,2, \cdots, n, \xi \in$ $\Gamma\left(L^{2}\left(\mathbb{R}_{+}, \mathbf{k}\right)\right)$ and disjoint intervals $\left[s_{k}, t_{k}\right)$,

(i) $\lim _{\underline{s} \rightarrow \underline{t}}\left\langle\xi, \prod_{k=1}^{n} M\left(s_{k}, t_{k}, u_{k}, v_{k}\right) \quad \boldsymbol{e}(0)\right\rangle=0$,

where $M\left(s_{k}, t_{k}, u_{k}, v_{k}\right)=\frac{\left(V_{s_{k}, t_{k}}-1\right)}{t_{k}-s_{k}}\left(u_{k}, v_{k}\right)-\left\langle u_{k}, G v_{k}\right\rangle-\gamma\left(s_{k}, t_{k}, u_{k}, v_{k}\right)$ and $\lim _{\underline{s} \rightarrow \underline{t}}$ means $s_{k}$ tends to $t_{k}$ for each $k$.

(ii) $\lim _{\underline{s} \rightarrow \underline{t}}\left\langle\xi, \otimes_{k=1}^{n} \gamma\left(s_{k}, t_{k}, u_{k}, v_{k}\right)\right\rangle=\left\langle\xi^{(n)}\left(t_{1}, t_{2}, \cdots, t_{n}\right), \eta\left(u_{1}, v_{1}\right) \otimes \cdots \otimes \eta\left(u_{n}\right.\right.$, $\left.\left.v_{n}\right)\right\rangle^{-}$

Proof. (i) First note that $M(s, t, u, v) \mathbf{e}(0)=\zeta(s, t, u, v)+\varsigma(s, t, u, v)$. So by the above observations $\{M(s, t, u, v) \mathbf{e}(0)\}$ is uniformly bounded in $s, t$ and $\lim _{s \rightarrow t}\langle\mathbf{e}(f), M(s, t, u, v) \mathbf{e}(0)\rangle=0, \forall f \in L^{2}\left(\mathbb{R}_{+}, \mathbf{k}\right)$. Since the intervals $\left[s_{k}, t_{k}\right)$ 's are disjoint for different $k$ 's,

$$
\left\langle\mathbf{e}(f), \prod_{k=1}^{n} M\left(s_{k}, t_{k}, u_{k}, v_{k}\right) \mathbf{e}(0)\right\rangle=\prod_{k=1}^{n}\left\langle\mathbf{e}\left(f_{\left[s_{k}, t_{k}\right)}\right), M\left(s_{k}, t_{k}, u_{k}, v_{k}\right) \mathbf{e}(0)\right\rangle
$$

and thus

$$
\lim _{\underline{s} \rightarrow \underline{t}}\left\langle\mathbf{e}(f), \prod_{k=1}^{n} M\left(s_{k}, t_{k}, u_{k}, v_{k}\right) \mathbf{e}(0)\right\rangle=0 .
$$


By Lemma 9.2 , the vector $\prod_{k=1}^{n} M\left(s_{k}, t_{k}, u_{k}, v_{k}\right) \mathbf{e}(0)$ is uniformly bounded in $s_{k}, t_{k}$ and the convergence can be extended to Fock Space.

(ii) It can be proved similarly as part (iii) of the previous Lemma.

Lemma 9.4. Let $\xi \in \Gamma$ be such that

$$
\langle\xi, \zeta\rangle=0, \forall \zeta \in \mathcal{S}^{\prime}
$$

Then

(i) $\xi^{(0)}=0$.

(ii) $\xi^{(1)}(t)=0$, for a.e. $t \in[0, \tau]$.

(iii) For any $n \geq 0, \xi^{(n)}(\underline{t})=0$, for a.e. $\underline{t} \in \Sigma_{n}: t_{i} \leq \tau$.

(iv) The set $\mathcal{S}^{\prime}$ is total in the Fock space $\Gamma$.

Proof. (i) For any $s \geq 0, V_{s, s}=1_{\mathbf{h} \otimes \Gamma}$ so in particular (9.4) gives, for any $u, v \in \mathbf{h}$

$$
0=\left\langle\xi, V_{s, s}(u, v) \mathbf{e}(0)\right\rangle=\langle u, v\rangle \overline{\xi^{(0)}}
$$

and hence $\xi^{(0)}=0$.

(ii) By (9.4), $\left\langle\xi,\left[V_{s, t}-1\right](u, v) \mathbf{e}(0)\right\rangle=0$ for any $0 \leq s<t \leq \tau<\infty, u, v \in \mathbf{h}$. By HP equation (8.2) and Lemma 9.1 we have

$$
\begin{aligned}
& 0=\lim _{s \rightarrow t} \frac{1}{t-s}\left\langle\xi,\left[V_{s, t}-1\right](u, v) \mathbf{e}(0)\right\rangle \\
& =\sum_{j \geq 1}\left\langle u, L_{j} v\right\rangle \overline{\xi_{j}^{(1)}(t)}=\sum_{j \geq 1} \eta_{j}(u, v) \overline{\xi_{j}^{(1)}(t)} .
\end{aligned}
$$

So $\left\langle\xi^{(1)}(t), \eta(u, v)\right\rangle=0, \forall u, v \in \mathbf{h}$. Since $\{\eta(u, v): u, v \in \mathbf{h}\}$ is total in $\mathbf{k}$ it follows that $\xi^{(1)}(t)=0$ for $0 \leq t \leq \tau$.

(iii) We prove this by induction. The result is already proved for $n=0,1$. For $n \geq 2$, assume as induction hypothesis that for all $m \leq n-1, \xi^{(m)}(\underline{t})=0$, for a.e. $\underline{t} \in \Sigma_{m}: t_{i} \leq \tau, i=1,2, \cdots, m$. We now show that $\xi^{(n)}(\underline{t})=0$, for a.e. $\underline{t} \in \Sigma_{n}: t_{i} \leq \tau$. 
Let $0 \leq s_{1}<t_{1} \leq s_{2}<t_{2}<\ldots<s_{n}<t_{n} \leq \tau$ and $u_{i}, v_{i} \in \mathbf{h}: i=$ $1,2 \cdots, n$. By $(9.4)$ and part (i) we have

$$
\left\langle\xi, \prod_{k=1}^{n} \frac{\left(V_{s_{k}, t_{k}}-1\right)}{t_{k}-s_{k}}\left(u_{k}, v_{k}\right) \mathbf{e}(0)\right\rangle=0 .
$$

Thus

$$
\begin{aligned}
& 0=\lim _{\underline{s} \rightarrow \underline{t}}\left\langle\xi, \prod_{k=1}^{n} \frac{\left(V_{s_{k}, t_{k}}-1\right)}{t_{k}-s_{k}}\left(u_{k}, v_{k}\right) \mathbf{e}(0)\right\rangle \\
& =\lim _{\underline{s} \rightarrow \underline{t}}\left\langle\xi, \prod_{k=1}^{n}\left\{M\left(s_{k}, t_{k}, u_{k}, v_{k}\right)+\left\langle u_{k}, G v_{k}\right\rangle+\gamma\left(s_{k}, t_{k}, u_{k}, v_{k}\right)\right\} \mathbf{e}(0)\right\rangle .
\end{aligned}
$$

Let $P, Q, R$ and $P^{\prime}, R^{\prime}$ be two sets of disjoint partitions of $\{1,2, \cdots, n\}$ such that $Q$ and $R$ are non empty. We write $|S|$ for the cardinality of set $S$. Then by Lemma 9.3 (ii) the right hand side of (9.5) is equal to

$$
\begin{aligned}
& \sum_{P^{\prime}, R^{\prime}}\left\langle\xi^{\left(\left|R^{\prime}\right|\right)}\left(t_{r_{1}^{\prime}}, \cdots, t_{r_{\left|R^{\prime}\right|}^{\prime}}\right), \otimes_{k \in R^{\prime}} \eta\left(u_{k}, v_{k}\right)\right\rangle \prod_{k \in P^{\prime}}\left\langle u_{k}, G v_{k}\right\rangle \\
& +\lim _{\underline{s} \rightarrow \underline{t}} \sum_{P, Q, R}\left\langle\xi, \prod_{k \in P}\left\langle u_{k}, G v_{k}\right\rangle \prod_{k \in Q}\left\{M\left(s_{k}, t_{k}, u_{k}, v_{k}\right)\right\} \prod_{k \in R}\left\{\gamma\left(s_{k}, t_{k}, u_{k}, v_{k}\right)\right\} \mathbf{e}(0)\right\rangle .
\end{aligned}
$$

Thus by the induction hypothesis,

(9.6)

$$
\begin{aligned}
& 0=\left\langle\xi^{(n)}\left(t_{1}, t_{2}, \cdots, t_{n}\right), \eta\left(u_{1}, v_{1}\right) \otimes \cdots \otimes \eta\left(u_{n}, v_{n}\right)\right\rangle \\
& +\lim _{\underline{s} \rightarrow \underline{t}} \sum_{P, Q, R}\left\langle\xi, \prod_{k \in P}\left\langle u_{k}, G v_{k}\right\rangle \prod_{k \in Q}\left\{M\left(s_{k}, t_{k}, u_{k}, v_{k}\right)\right\} \prod_{k \in R}\left\{\gamma\left(s_{k}, t_{k}, u_{k}, v_{k}\right)\right\} \mathbf{e}(0)\right\rangle .
\end{aligned}
$$

We claim that the second term in (9.6) vanishes. To prove this claim, it is enough to show that for any two non empty disjoint subsets $Q \equiv\left\{q_{1}, q_{2}, \cdots\right.$, $\left.q_{|Q|}\right\}, R \equiv\left\{r_{1}, r_{2}, \cdots, r_{|R|}\right\}$ of $\{1,2, \cdots, n\}$,

$$
\lim _{\underline{s} \rightarrow \underline{t}}\left\langle\xi, \prod_{q \in Q}\left\{M\left(s_{q}, t_{q}, u_{q}, v_{q}\right)\right\} \prod_{r \in R}\left\{\gamma\left(s_{r}, t_{r}, u_{r}, v_{r}\right)\right\} \quad \mathbf{e}(0)\right\rangle=0 .
$$


Writing $\psi$ for the vector $\prod_{q \in Q}\left\{M\left(s_{q}, t_{q}, u_{q}, v_{q}\right)\right\} \mathbf{e}(0)$, we have

$$
\begin{aligned}
\left\langle\xi, \prod_{q \in Q}\left\{M\left(s_{q}, t_{q}, u_{q}, v_{q}\right)\right\} \prod_{r \in R}\left\{\gamma\left(s_{r}, t_{r}, u_{r}, v_{r}\right)\right\} \mathbf{e}(0)\right\rangle \\
=\left\langle\xi, \psi \otimes \otimes_{r \in R} \frac{1_{\left[s_{r}, t_{r}\right]} \eta\left(u_{r}, v_{r}\right)}{t_{r}-s_{r}}\right\rangle \\
=\left\langle\xi, \psi \otimes \otimes_{r \in R} \frac{1_{\left[s_{r}, t_{r}\right]} \eta\left(u_{r}, v_{r}\right)}{t_{r}-s_{r}}\right\rangle \\
=\sum_{l \geq|R|}\left\langle\xi^{(l)}, \psi^{(l-|R|)} \otimes \otimes_{r \in R} \frac{1_{\left[s_{r}, t_{r}\right]} \eta\left(u_{r}, v_{r}\right)}{t_{r}-s_{r}}\right\rangle \\
=\left\langle\sum_{l \geq|R|}\left\langle\left\langle\xi^{(l)}, \psi^{(l-|R|)}\right\rangle\right\rangle, \otimes_{r \in R} \frac{1_{\left[s_{r}, t_{r}\right]} \eta\left(u_{r}, v_{r}\right)}{t_{r}-s_{r}}\right\rangle .
\end{aligned}
$$

Here $\left\langle\left\langle\xi^{(l)}, \psi^{(l-|R|)}\right\rangle\right\rangle \in L^{2}\left(\mathbf{R}_{+}, \mathbf{k}\right)^{\otimes|R|}$ is defined as in (2.1) by

$$
\begin{aligned}
\langle & \left.\left\langle\left\langle\xi^{(l)}, \psi^{(l-|R|)}\right\rangle\right\rangle, \rho^{(|R|)}\right\rangle=\left\langle\xi^{(l)}, \psi^{(l-|R|)} \otimes \rho^{(|R|)}\right\rangle \\
= & \int_{\Sigma_{l}}\left\langle\xi^{(l)}\left(x_{1}, x_{2}, \cdots, x_{l}\right), \psi^{(l-|R|)}\left(x_{1}, x_{2}, \cdots, x_{l-|R|}\right)\right. \\
& \left.\otimes \rho^{(|R|)}\left(x_{l-|R|+1}, \cdots, x_{l}\right)\right\rangle_{\mathbf{k}^{\otimes l}} d x
\end{aligned}
$$

for any $\rho^{(|R|)} \in L^{2}\left(\mathbf{R}_{+}, \mathbf{k}\right)^{\otimes|R|}$.

By Lemma 9.3 (i),

$$
\lim _{s_{q} \rightarrow t_{q}}\left\langle\xi, \prod_{q \in Q}\left\{M\left(s_{q}, t_{q}, u_{q}, v_{q}\right)\right\} \prod_{r \in R}\left\{\gamma\left(s_{r}, t_{r}, u_{r}, v_{r}\right)\right\} \quad \mathbf{e}(0)\right\rangle=0 .
$$

However, we need to prove (9.7) where the limit $\underline{s} \rightarrow \underline{t}$ has to be in arbitrary order. On the other hand, by (9.8) and (9.9) we get

$$
\begin{aligned}
& \lim _{s_{q} \rightarrow t_{q}} \lim _{s_{r} \rightarrow t_{r}}\left\langle\xi, \prod_{q \in Q}\left\{M\left(s_{q}, t_{q}, u_{q}, v_{q}\right)\right\} \prod_{r \in R}\left\{\gamma\left(s_{r}, t_{r}, u_{r}, v_{r}\right)\right\} \mathbf{e}(0)\right\rangle \\
= & \lim _{s_{q} \rightarrow t_{q}} \lim _{s_{r} \rightarrow t_{r}}\left\langle\sum_{l \geq|R|}\left\langle\left\langle\xi^{(l)}, \psi^{(l-|R|)}\right\rangle\right\rangle, \otimes_{r \in R} \frac{1_{\left[s_{r}, t_{r}\right]} \eta\left(u_{r}, v_{r}\right)}{t_{r}-s_{r}}\right\rangle \\
= & \lim _{s_{q} \rightarrow t_{q}} \lim _{s_{r} \rightarrow t_{r}}\left\langle\int _ { \Sigma _ { | R | } } \left\langle\left[\sum_{l \geq|R|}\left\langle\left\langle\xi^{(l)}, \psi^{(l-|R|)}\right\rangle\right\rangle\right]\left(x_{1}, x_{2}, \cdots, x_{|R|}\right),\right.\right. \\
= & \lim _{s_{q} \rightarrow t_{q}}\left\langle\sum_{l \geq|R|}\left\langle\frac{1_{\left[s_{r}, t_{r}\right]}\left(x_{r}\right) \eta\left(u_{r}, v_{r}\right)}{t_{r}-s_{r}}\right\rangle d x\right.
\end{aligned}
$$


for almost all $\underline{t} \in \Sigma_{|R|}$. We fix $\underline{t} \in \Sigma_{|R|}$ and define families of vectors $\widetilde{\xi}^{(l)}: l \geq 0$ in $L^{2}\left(\mathbb{R}_{+}, \mathbf{k}\right)^{\otimes l}$ by

$$
\begin{aligned}
& \widetilde{\xi}^{(0)}=\left\langle\xi^{(|R|)}\left(t_{r_{1}}, \cdots, t_{r_{|R|}}\right), \otimes_{r \in R} \eta\left(u_{r}, v_{r}\right)\right\rangle \in \mathbb{C} \\
& \widetilde{\xi}^{(l)}\left(x_{1}, x_{2}, \cdots, x_{l}\right)=\left\langle\left\langle\xi^{(|R|+l)}\left(x_{1}, \cdots, x_{l}, t_{r_{1}}, \cdots, t_{r_{|R|}}\right), \otimes_{r \in R} \eta\left(u_{r}, v_{r}\right)\right\rangle\right\rangle,
\end{aligned}
$$

which defines a Fock space vector $\widetilde{\xi}$. Therefore, from (9.11), we get that

$$
\begin{aligned}
& \lim _{s_{q} \rightarrow t_{q}} \lim _{s_{r} \rightarrow t_{r}}\left\langle\xi, \prod_{q \in Q}\left\{M\left(s_{q}, t_{q}, u_{q}, v_{q}\right)\right\} \prod_{r \in R}\left\{\gamma\left(s_{r}, t_{r}, u_{r}, v_{r}\right)\right\} \mathbf{e}(0)\right\rangle=\lim _{s_{q} \rightarrow t_{q}}\langle\widetilde{\xi}, \psi\rangle \\
& =\lim _{s_{q} \rightarrow t_{q}}\left\langle\widetilde{\xi},\left[\prod_{q \in Q} M\left(s_{q}, t_{q}, u_{q}, v_{q}\right)\right] \mathbf{e}(0)\right\rangle,
\end{aligned}
$$

which is equal to 0 by Lemma 9.3 (a). Thus from (9.6) we get that

$$
\left\langle\xi^{(n)}\left(t_{1}, t_{2}, \cdots, t_{n}\right), \eta\left(u_{1}, v_{1}\right) \otimes \cdots \otimes \eta\left(u_{n}, v_{n}\right)\right\rangle=0 .
$$

Since $\{\eta(u, v): u, v \in \mathbf{h}\}$ is total in $\mathbf{k}$, it follows that $\xi^{(n)}\left(t_{1}, t_{2}, \cdots, t_{n}\right)=0$ for almost every $\left(t_{1}, t_{2}, \cdots, t_{n}\right) \in \Sigma_{n}: t_{k} \leq \tau$.

(iv) Since $\tau \geq 0$ is arbitrary $\xi^{(n)}=0 \in L^{2}\left(\mathbb{R}_{+}, \mathbf{k}\right)^{\otimes n}: n \geq 0$ and hence $\xi=0$. This proves the totality of $\mathcal{S}^{\prime}$ in $\Gamma$.

\section{$\S 10$. Unitary Equivalence}

Here we shall show that the unitary evolution $\left\{U_{s, t}\right\}$ on $\mathbf{h} \otimes \mathcal{H}$ is unitarily equivalent to the HP flow $\left\{V_{s, t}\right\}$ on $\mathbf{h} \otimes \Gamma\left(L^{2}\left(\mathbb{R}_{+}, \mathbf{k}\right)\right)$ discussed above. Let us recall that the subset $\mathcal{S}=\left\{\xi=U_{\underline{s}, \underline{t}}(\underline{u}, \underline{v}) \Omega:=U_{s_{1}, t_{1}}\left(u_{1}, v_{1}\right) \cdots U_{s_{n}, t_{n}}\left(u_{n}, v_{n}\right) \Omega\right.$ : $\underline{s}=\left(s_{1}, s_{2}, \cdots, s_{n}\right), \underline{t}=\left(t_{1}, t_{2}, \cdots, t_{n}\right): 0 \leq s_{1} \leq t_{1} \leq s_{2} \leq \ldots \leq s_{n} \leq t_{n}<$ $\left.\infty, n \geq 1, \underline{u}=\otimes_{i=1}^{n} u_{i}, \underline{v}=\otimes_{i=1}^{n} v_{i} \in \mathbf{h}^{\otimes n}\right\}$ is total in $\mathcal{H}$ and the subset $\mathcal{S}^{\prime}:=\left\{\zeta=V_{\underline{s}, \underline{t}}(\underline{u}, \underline{v}) \mathbf{e}(0):=V_{s_{1}, t_{1}}\left(u_{1}, v_{1}\right) \cdots V_{s_{n}, t_{n}}\left(u_{n}, v_{n}\right) \mathbf{e}(0):\right.$ $\left.\underline{u}, \underline{v} \in \mathbf{h}^{\otimes n}, \underline{s}=\left(s_{1}, s_{2}, \cdots, s_{n}\right), \underline{t}=\left(t_{1}, t_{2}, \cdots, t_{n}\right)\right\}$ is total in $\Gamma$.

Lemma 10.1. Let $U_{\underline{s}, \underline{t}}(\underline{u}, \underline{v}) \Omega, \quad U_{\underline{s}^{\prime}, \underline{t}^{\prime}}(\underline{p}, \underline{w}) \Omega \in \mathcal{S}$.

Then there exist an integer $m \geq 1, \underline{a}=\left(a_{1}, a_{2}, \cdots, a_{m}\right), \underline{b}=\left(b_{1}, b_{2}, \cdots, b_{m}\right)$ : $0 \leq a_{1} \leq b_{1} \leq a_{2} \leq \ldots \leq a_{m} \leq b_{m}<\infty$, an ordered partition $R_{1} \cup R_{2} \cup R_{3}=$ $\{1,2, \cdots, m\}$ with $\left|R_{i}\right|=m_{i}$ and a family of vectors $x_{k_{l}}, y_{k_{l}} \in \mathbf{h}, k_{l} \geq 1: l=$ $1,2, \cdots, m_{1}+m_{2}$ and $g_{k_{l}}, h_{k_{l}} \in \mathbf{h}, k_{l} \geq 1: l=1,2, \cdots, m_{2}+m_{3}$ such that

$$
U_{\underline{s}, \underline{t}}(\underline{u}, \underline{v})=\sum_{\underline{k}} \prod_{l \in R_{1} \cup R_{2}} U_{a_{l}, b_{l}}\left(x_{k_{l}}, y_{k_{l}}\right)
$$




$$
U_{{\underline{s^{\prime}}}^{\prime}, \underline{t}^{\prime}}(\underline{p}, \underline{w})=\sum_{\underline{k}} \prod_{l \in R_{2} \cup R_{3}} U_{a_{l}, b_{l}}\left(g_{k_{l}}, h_{k_{l}}\right) .
$$

Proof. This follows from the evolution hypothesis of the family of unitary operators $\left\{U_{s, t}\right\}$.

Remark 10.2. Since the family of unitaries $\left\{V_{s, t}\right\}$ on $\mathbf{h} \otimes \Gamma$, enjoy all the properties satisfied by the family of unitaries $\left\{U_{s, t}\right\}$ on $\mathbf{h} \otimes \mathcal{H}$, the above Lemma also hold if we replace $U_{s, t}$ by $V_{s, t}$.

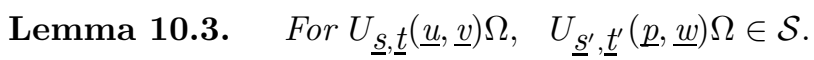

$$
\left\langle U_{\underline{s}, \underline{t}}(\underline{u}, \underline{v}) \Omega, U_{\underline{s^{\prime}}, \underline{t^{\prime}}}(\underline{p}, \underline{w}) \Omega\right\rangle=\left\langle V_{\underline{s}, \underline{t}}(\underline{u}, \underline{v}) \boldsymbol{e}(0), V_{\underline{s}^{\prime}, \underline{t}^{\prime}}(\underline{p}, \underline{w}) \boldsymbol{e}(0)\right\rangle .
$$

Proof. We have by previous Lemma and Assumption: A

$$
\begin{aligned}
& \left\langle U_{\underline{s}, \underline{t}}(\underline{u}, \underline{v}) \Omega, U_{\underline{S}^{\prime}, \underline{t}^{\prime}}(\underline{p}, \underline{w}) \Omega\right\rangle \\
& =\sum_{\underline{k}} \prod_{l \in R_{1}}\left\langle U_{b_{l}-a_{l}}\left(x_{k_{l}}, y_{k_{l}}\right) \Omega, \Omega\right\rangle \prod_{l \in R_{2}}\left\langle U_{b_{l}-a_{l}}\left(x_{k_{l}}, y_{k_{l}}\right) \Omega, U_{b_{l}-a_{l}}\left(g_{k_{l}}, h_{k_{l}}\right) \Omega\right\rangle \\
& \prod_{l \in R_{3}}\left\langle\Omega, U_{b_{l}-a_{l}}\left(g_{k_{l}}, h_{k_{l}}\right) \Omega\right\rangle \\
& =\sum_{\underline{k}} \prod_{l \in R_{1}}\left\langle T_{b_{l}-a_{l}} y_{k_{l}}, x_{k_{l}}\right\rangle \prod_{l \in R_{2}}\left\langle g_{k_{l}}, Z_{b_{l}-a_{l}}\left(\left|h_{k_{l}}><y_{k_{l}}\right|\right) x_{k_{l}}\right\rangle \\
& \prod_{l \in R_{3}}\left\langle g_{k_{l}}, T_{b_{l}-a_{l}} h_{k_{l}}\right\rangle \\
& =\sum_{\underline{k}} \prod_{l \in R_{1}}\left\langle V_{b_{l}-a_{l}}\left(x_{k_{l}}, y_{k_{l}}\right) \mathbf{e}(0), \mathbf{e}(0)\right\rangle \\
& \prod_{l \in R_{2}}\left\langle V_{b_{l}-a_{l}}\left(x_{k_{l}}, y_{k_{l}}\right) \mathbf{e}(0), V_{b_{l}-a_{l}}\left(g_{k_{l}}, h_{k_{l}}\right) \mathbf{e}(0)\right\rangle \\
& \prod_{l \in R_{3}}\left\langle\mathbf{e}(0), V_{b_{l}-a_{l}}\left(g_{k_{l}}, h_{k_{l}}\right) \mathbf{e}(0)\right\rangle .
\end{aligned}
$$

Now, the above quantity is equal to $\left\langle V_{\underline{s}, \underline{t}}(\underline{u}, \underline{v}) \mathbf{e}(0), V_{\underline{S}^{\prime}, \underline{t^{\prime}}}(\underline{p}, \underline{w}) \mathbf{e}(0)\right\rangle$ by Remark (10.2). 
Theorem 10.4. There exists a unitary isomorphism $\Xi: \mathbf{h} \otimes \mathcal{H} \rightarrow \mathbf{h} \otimes \Gamma$ such that

$$
U_{t}=\Xi^{*} V_{t} \Xi, \forall t \geq 0
$$

Proof. Let us define a map $\Xi: \mathcal{H} \rightarrow \Gamma$ by setting, for any $\xi=U_{\underline{s}, \underline{t}}(\underline{u}, \underline{v}) \Omega \in$ $\left.\mathcal{S}, \quad \Xi \xi:=V_{\underline{s}, t} \underline{u}, \underline{v}\right) \mathbf{e}(0) \in \mathcal{S}^{\prime}$ and then extending linearly. So by definition and by the totality of $\mathcal{S}^{\prime}$, the range of $\Xi$ is dense in $\Gamma$. To see that $\Xi$ is a unitary operator from $\mathcal{H}$ to $\Gamma$ it is enough to note that

$$
\left\langle\Xi \xi, \Xi \xi^{\prime}\right\rangle=\left\langle\xi, \xi^{\prime}\right\rangle, \forall \xi, \xi^{\prime} \in \mathcal{S}
$$

which is already proved in the previous Lemma.

Now consider the ampliated unitary operator $1_{\mathbf{h}} \otimes \Xi$ from $\mathbf{h} \otimes \mathcal{H}$ to $\mathbf{h} \otimes \Gamma$ and denote it by the same symbol $\Xi$. In order to prove (10.4) it is enough to show that

$$
\left\langle u \otimes \xi, U_{t} v \otimes \xi^{\prime}\right\rangle=\left\langle\Xi(u \otimes \xi), V_{t} \Xi\left(v \otimes \xi^{\prime}\right)\right\rangle, \forall u, v \in \mathbf{h}, \xi, \xi^{\prime} \in \mathcal{S} .
$$

Note that $\Xi U_{t}(u, v) \xi^{\prime}=V_{t}(u, v) \Xi \xi^{\prime}$. Now by unitarity of $\Xi$, we have

$$
\begin{aligned}
& \left\langle u \otimes \xi, U_{t} v \otimes \xi^{\prime}\right\rangle=\left\langle\xi, U_{t}(u, v) \xi^{\prime}\right\rangle=\left\langle\Xi \xi, \Xi U_{t}(u, v) \xi^{\prime}\right\rangle \\
& =\left\langle\Xi \xi, V_{t}(u, v) \Xi \xi^{\prime}\right\rangle=\left\langle u \otimes \Xi \xi, V_{t} v \otimes \Xi \xi^{\prime}\right\rangle=\left\langle\Xi(u \otimes \xi), V_{t} \Xi\left(v \otimes \xi^{\prime}\right)\right\rangle .
\end{aligned}
$$

Thus proof complete.

\section{Acknowledgements}

The first author acknowledges National Board for Higher Mathematics, DAE, India for research support. The last author acknowledges the partial support of Bhatnagar Fellowship Project of CSIR, India. A portion of this work was completed at Delhi Centre of Indian Statistical Institute. All three authors have benefited from the DST-DAAD (Indo-German) Exchange Programme at the early stage of this work.

\section{References}

[1] L. Accardi, J.-L. Journé and J. M. Lindsay, On multi-dimensional Markovian cocycles, in Quantum probability and applications, IV (Rome, 1987), 59-67, Lecture Notes in Math., 1396, Springer, Berlin, 1989.

[2] I. M. Gel'fand and N. Ya. Vilenkin, Generalized functions. Vol. 4, Applications of harmonic analysis, Translated by Amiel Feinstein, Academic Press, New York, 1964. 
[3] K. B. Sinha and D. Goswami, Quantum stochastic processes and noncommutative geometry, Cambridge Tracts in Mathmatics, 169, Cambridge Univ. Press, Cambridge, 2007.

[4] R. L. Hudson and J. M. Lindsay, On characterizing quantum stochastic evolutions, Math. Proc. Cambridge Philos. Soc. 102 (1987), no. 2, 363-369.

[5] J.-L. Journé, Structure des cocycles markoviens sur l'espace de Fock, Probab. Theory Related Fields 75 (1987), no. 2, 291-316.

[6] R. L. Hudson and K. R. Parthasarathy, Quantum Ito's formula and stochastic evolutions, Comm. Math. Phys. 93 (1984), no. 3, 301-323.

[7] Stochastic dilations of uniformly continuous completely positive semigroups, Acta Appl. Math. 2 (1984), no. 3-4, 353-378.

[8] J. M. Lindsay and A. G. Skalski, Quantum stochastic convolution cocycles. I, Ann. Inst. H. Poincaré Probab. Statist. 41 (2005), no. 3, 581-604.

[9] , Quantum stochastic convolution cocycles. II, Comm. Math. Phys. 280 (2008), no. 3, 575-610.

[10] J. M. Lindsay and S. J. Wills, Markovian cocycles on operator algebras adapted to a Fock filtration, J. Funct. Anal. 178 (2000), no. 2, 269-305.

[11] Construction of some quantum stochastic operator cocycles by the semigroup method, Proc. Indian Acad. Sci. Math. Sci. 116 (2006), no. 4, 519-529.

[12] L. Sahu and K. B. Sinha, Characterization of unitary processes with independent and stationary increments. Submitted to Ann. Inst. Henri Poincaré, Probabilités et Statistiqués, Available at: http://lanl.arxiv.org/abs/0804.1948

[13] P.-A. Meyer, Quantum probability for probabilists, Lecture Notes in Math., 1538, Springer, Berlin, 1993.

[14] A. Mohari and K. B. Sinha, Quantum stochastic flows with infinite degrees of freedom and countable state Markov processes, Sankhyā Ser. A 52 (1990), no. 1, 43-57.

[15] Stochastic dilation of minimal quantum dynamical semigroup, Proc. Indian Acad. Sci. Math. Sci. 102 (1992), no. 3, 159-173.

[16] K. R. Parthasarathy, An introduction to quantum stochastic calculus, Monographs in Mathematics, 85, Birkhäuser, Basel, 1992.

[17] K. R. Parthasarathy and K. B. Sinha, Stochastic integral representation of bounded quantum martingales in Fock space, J. Funct. Anal. 67 (1986), no. 1, 126-151.

[18] M. Schürmann, Noncommutative stochastic processes with independent and stationary increments satisfy quantum stochastic differential equations, Probab. Theory Related Fields 84 (1990), no. 4, 473-490.

[19] M. Schürmann, White noise on bialgebras, Lecture Notes in Math., 1544, Springer, Berlin, 1993. 